\title{
Determinación del Nivel de Precios y la Dinámica \\ Inflacionaria en México
}

\author{
Daniel G. Garcés Díaz*
}

Diciembre de 1999

Documento de Investigación No. 9907

\section{Dirección General de Investigación Económica}

\author{
BANCO DE MEXICO
}

\footnotetext{
*El autor agradece las sugerencias y comentarios de Alejandro Werner. Otras personas que leyeron el documento e hicieron valiosas propuestas para mejorarlo son Rafael GómezTagle, Sara Castellanos, Alejandro Pérez-López, Adalberto González y Oscar Sánchez. Rocío Elizondo colaboró en esta investigación de manera muy eficaz desde el comienzo. Todas las personas mencionadas no tienen parte en cualquier omisión o error que el documento pueda contener. Las opiniones vertidas en el documento reflejan exclusivamente el punto de vista del autor y no necesariamente las del Banco de México.
} 


\title{
Determinación del Nivel de Precios y la Dinámica \\ Inflacionaria en México
}

Daniel G. Garcés Díaz

Diciembre de 1999

Documento de Investigación No. 9907

\begin{abstract}
Resumen
En este trabajo se presenta un modelo econométrico para explicar el comportamiento de la inflación en México durante el período 1985 a 1998. Los hallazgos del documento formalizan, clarifican y cuantifican varias ideas sobre el tema mediante la utilización de métodos estadísticos apropiados. Se demuestra que los determinantes más importantes del nivel de precios doméstico, salarios y precios externos, tienen con aquél una única relación de equilibrio que se interpreta como una ecuación de precios de largo plazo y homogénea de grado uno. Posteriormente, se deriva un modelo para la dinámica de ajuste. A partir de dicho modelo se muestran las respuestas de la tasa de inflación a través del tiempo frente a los ajustes en los salarios, el tipo de cambio, los precios públicos y la desviación del producto de su nivel potencial.
\end{abstract}




\section{Introducción}

El entendimiento de la dinámica inflacionaria en México es un asunto de enorme importancia para los agentes económicos y las autoridades financieras. El presente trabajo es un aporte al entendimiento empírico de este fenómeno. De entre un menú amplio de opciones teóricas y econométricas para estudiar los distintos aspectos del fenómeno inflacionario, elegimos un enfoque bastante general y robusto. Para no desviar demasiado la atención de las implicaciones económicas del modelo, varios detalles técnicos se remiten a apéndices en donde los lectores especializados pueden juzgar la confiabilidad del análisis estadístico.

En primer lugar, en el documento se describen las series utilizadas y se estudian brevemente sus propiedades estadísticas individuales. A continuación, se describe el marco conceptual utilizado, el cual es una ecuación estándar de precios en una economía pequeña y abierta, la cual dice que el nivel precios internos es una función Cobb-Douglas de los salarios y los precios externos (cuyo componente más variable en el caso de México es el tipo de cambio nominal). La estimación de las elasticidades respectivas en este modelo es el primer paso del trabajo econométrico.

Para obtener estos estimados se aplica una técnica llamada análisis de cointegración, la cual parece apropiada debido a las propiedades estadísticas de las series. La ecuación de precios así estimada debe cumplirse en el largo plazo ${ }^{1}$ y no necesariamente en el corto.

Los valores encontrados para estas elasticidades fueron de 0.36 para los salarios y de 0.63 para los precios externos (nótese que la suma de las elasticidades es básicamente igual a la unidad), aunque posiblemente estos valores se alteraron ligeramente después de 1994 con la adopción del régimen de tipo de cambio flexible. Esta posibilidad, a pesar de su importancia, se deja como material para otro documento. La interpretación de estas elasticidades es la siguiente. Si ocurre un incremento de $10 \%$ en los salarios, en el largo plazo el nivel de precios interno debe incrementarse en $3.6 \%$, mientras que un incremento similar en precios externos hará que el nivel de precios suba $6.3 \%$.

Cuando la relación se cumple exactamente (es decir cuando el logaritmo del nivel de precios es igual a una constante más 0.36 por el nivel de salarios más 0.63 por los precios externos), se dice que el sistema se encuentra en su equilibrio de largo plazo. Se muestra gráficamente que el ajuste de la ecuación estimada a los datos reales es bastante bueno, pero que en general las diferencias entre las series estimada y ajustada son diferentes de cero. Estas desviaciones (llamadas errores de equilibrio) tienen implicaciones muy importantes para la dinámica inflacionaria. Cuando el nivel de precios de equilibrio se encuentra por arriba (abajo) del nivel observado del índice de precios existe una presión inflacionaria (deflacionaria). El tiempo en el cual dicho error de equilibrio tarda en desaparecer es lo que aquí se denomina "largo plazo."

Para tener una idea más precisa de cómo se comporta la inflación (que es la única variable que se ajusta en el corto plazo para restablecer el equilibrio

\footnotetext{
${ }^{1} \mathrm{La}$ expresión "largo plazo" tiene un significado preciso en este documento y, como se mostrará más adelante, se refiere a un periodo
} 
de largo plazo) se deriva un modelo para el comportamiento de esta variable. El modelo para la inflación es una regresión típica que tiene como variables explicatorias un rezago de ella misma (lo cual resulta de cierto grado de inercia), los errores de equilibrio (que, como se indicó anteriormente, señalan la presencia de presiones inflacionarias o deflacionarias), la tasa de cambio de salarios, precios externos, precios de bienes públicos, la brecha de producto y variables estacionales. Otras variables construidas a partir de los diferentes agregados monetarios, cambios en productividad y los salarios mínimos no agregan ningún poder explicativo al modelo.

Después de discutir la interpretación económica de los estimados de la ecuación de inflación, se llevan a cabo un conjunto de ejercicios de simulación que ilustran las propiedades dinámicas del modelo. Para tener una descripción más completa de la dinámica inflacionaria se estiman también, modelos simples para la tasa de cambio de los salarios y la brecha de producto. Se encuentra que la velocidad de convergencia del sistema ante un ajuste salarial es mayor que ante un ajuste al tipo de cambio.

Después de seis meses el ajuste salarial se ha transmitido en $66 \%$ mientras que el ajuste cambiario se transmite en solamente $40 \%$. En doce meses los porcentajes son $81 \%$ y $65 \%$, respectivamente. En dieciocho meses el ajuste salarial se ha transmitido en $89 \%$ contra $80 \%$ del ajuste al tipo de cambio. En ambos casos el tiempo necesario para que el ajuste se incorpore completamente al nivel de precios toma más de dos años.

Para poner estos resultados en perspectiva, se hace una comparación con el caso de un país de baja inflación. Se muestra que la velocidad a la cual un ajuste del tipo de cambio se transmite a los precios en Australia (donde las velocidades de ajuste a choques salariales y cambiarios son iguales) es mucho menor que la encontrada en el modelo para México.

También se llevaron a cabo ejercicios similares para los precios públicos y la brecha de producto. Estas variables tienen efectos de largo plazo sobre el nivel de precios a través de su influencia sobre los salarios.

El resto del documento está organizado de la siguiente manera. En la sección 2 se describen los datos utilizados. En la sección 3 se discute el orden de integración de las variables utilizadas. En la sección 4 se analizan las relaciones de largo plazo (cointegración) entre las variables. En la sección 5 se derivan los modelos de corrección de error y se analizan sus propiedades dinámicas. Finalmente, se ofrecen las conclusiones en la sección 6.

\section{Descripción de los Datos y los Símbolos Uti- lizados}

La frecuencia de todas las series consideradas es mensual y comienzan en 1980 para el Indice Nacional de Precios al Consumidor $(P)$, el índice de salarios y los precios externos y desde 1985 para el resto de las series. Los nombres de las variables en minúsculas representan logaritmos de las mismas (por ejemplo 
$p$ representa el logaritmo de $P$ ).

La letra $d$ antes de una variable es el operador de primera diferencia (esto es, $d x=\Delta x=(1-L) x=x-x_{t-1}$ donde $L$ es el operador de rezagos: $\left.L x=x_{t-1}\right)$. Por ejemplo, $d p=p_{t}-p_{t-1}$ es la tasa de inflación de $P$ de un mes a otro. La tasa de cambio de una variable del mes de un año al mismo mes del año anterior se representa como $d^{a} x=\left(1-L^{12}\right) x=x_{t}-x_{t-12}$. Así, $d^{a} p$ es la tasa de inflación anualizada. Esta última definición es muy útil para aislar los determinantes de largo plazo de los factores estacionales y de corto plazo.

La serie de mayor interés para esta investigación es el Indice Nacional de Precios al Consumidor $(P)$. También es interesante el modelaje de un índice que no incluya los precios de los bienes que están sujetos a control oficial o administrados por el sector público. Utilizamos un índice elaborado por Gamboa (1997) que incluye únicamente aquéllos bienes cuyos precios no están sujetos a control oficial $(P N C)$. El complemento de este índice es el de precios controlados $(P C) .^{2}$

Desafortunadamente, estos dos últimos índices comienzan recién en enero de 1985 lo que da apenas 14 años de longitud de muestra. Sin embargo, la longitud de la serie es mucho más importante para el análisis de integración de la serie que para el de cointegración. Si uno parte de la premisa de que las series no son $I(0)$ y tienen alguna relación de largo plazo entonces la longitud mínima de una serie necesaria para llevar a cabo un análisis de cointegración depende de la velocidad de ajuste del sistema. Más adelante se verá que 14 años es un lapso suficientemente bueno para realizar el análisis de cointegración de este documento. Esto resulta de la magnitud de la velocidad de ajuste en el modelo que aquí se estima.

La gráfica 1 muestra las variaciones mensuales porcentuales (o inflaciones) del Indice Nacional de Precios al Consumidor $P$ y del índice de precios no controlados $P N C$ ( $d p$ y $d p n c$, respectivamente). Se puede notar que las diferencias entre ambas se van reduciendo con el paso del tiempo hasta que a partir de 1995 son casi indistinguibles. Esto tiene que ver con la continua liberación de precios anteriormente controlados y con el aumento de la frecuencia de ajuste de los preciosque permanecen bajo control. El porcentaje de éstos en la canasta utilizada para elaborar el INPC llegó a ser de más de 40\%. Para finales de 1998 este porcentaje cayó a casi $13 \%{ }^{3}$

\footnotetext{
${ }^{2}$ Gamboa (1997) incluye en su definición de precios controlados o administrados a los precios de los bienes y servicios que produce el sector público, el de algunos bienes que reciben subsidio y tienen un fuerte impacto sobre el gasto de los consumidores así como el de otros bienes sobre los cuales pocos oferentes tienen considerable poder de mercado.

${ }^{3}$ Para una descripción detallada de este proceso ver Gamboa (1997).
} 


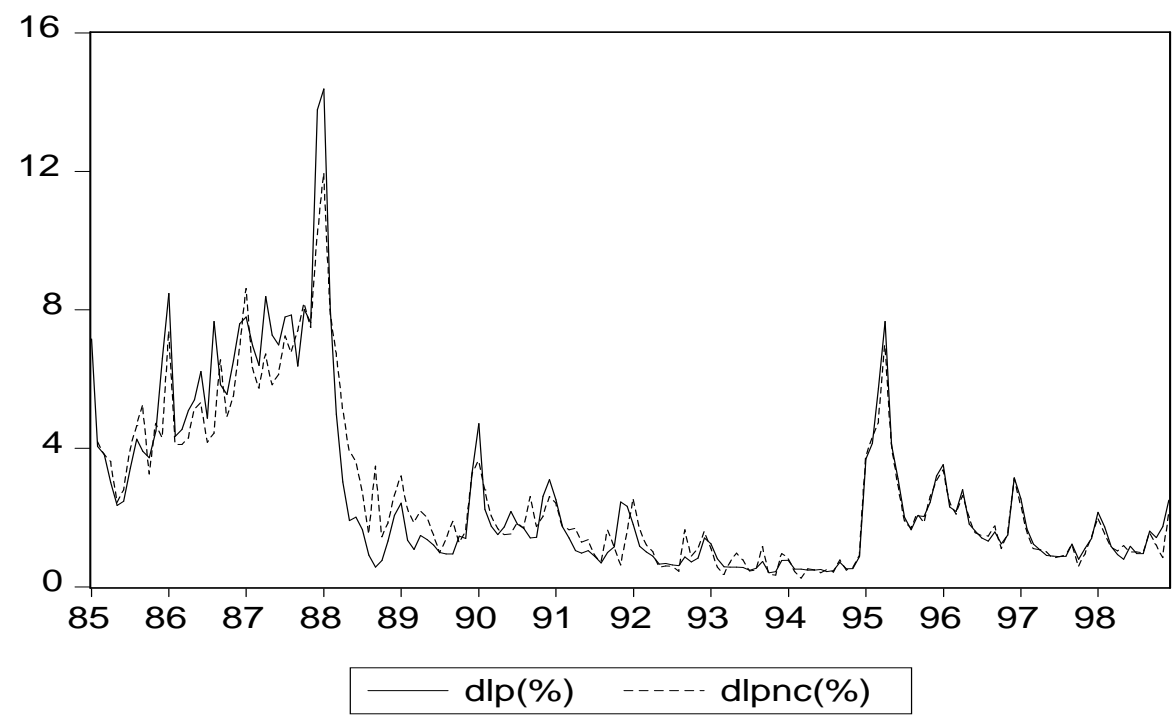

Gráfica 1: La Inflación del Indice Nacional de Precios al Consumidor (línea continua) La Inflación del Indice de Precios no Controlados (línea punteada).

La variable que representa los costos laborales nominales $(W)$ es la serie de remuneraciones salariales totales medias del sector manufacturero. Los precios externos $(E P E U)$ se construyeron con el índice de precios al consumidor para Estados Unidos y el tipo de cambio del peso respecto al dólar. Aunque se podría objetar que en lugar de esta definición de precios externos se debería usar un índice de precios de bienes importados, ${ }^{4}$ la definición aquí empleada es similar a la utilizada por Juselius (1992) ${ }^{5}$ además de que la inflación externa se da sobre los precios de todos los bienes comerciables y no solo los importados.

El tipo de cambio es la principal fuente de variación de $E P E U$ así que en el texto ambas variables se utilizan de modo indistinto. La gráfica 2 muestra a la inflación anualizada del Indice Nacional de Precios al Consumidor, $P$, la tasa de crecimiento anualizada de los salarios (esto es, $d^{a} w$ ) y la inflación externa anualizada (o $\left.d^{a} e p e u\right)$. La fuerte relación entre las tres variables es evidente y el modelo econométrico que se presenta está basado en ella.

\footnotetext{
${ }^{4}$ Como por ejemplo en De Brouwer y Ericsson (1988) en su modelo de inflación para Australia.

${ }^{5}$ Esta autora construye un modelo de inflación para la economía danesa utilizando los precios de Alemania (que es por mucho el principal socio comercial de Dinamarca) para representar los precios externos.
} 


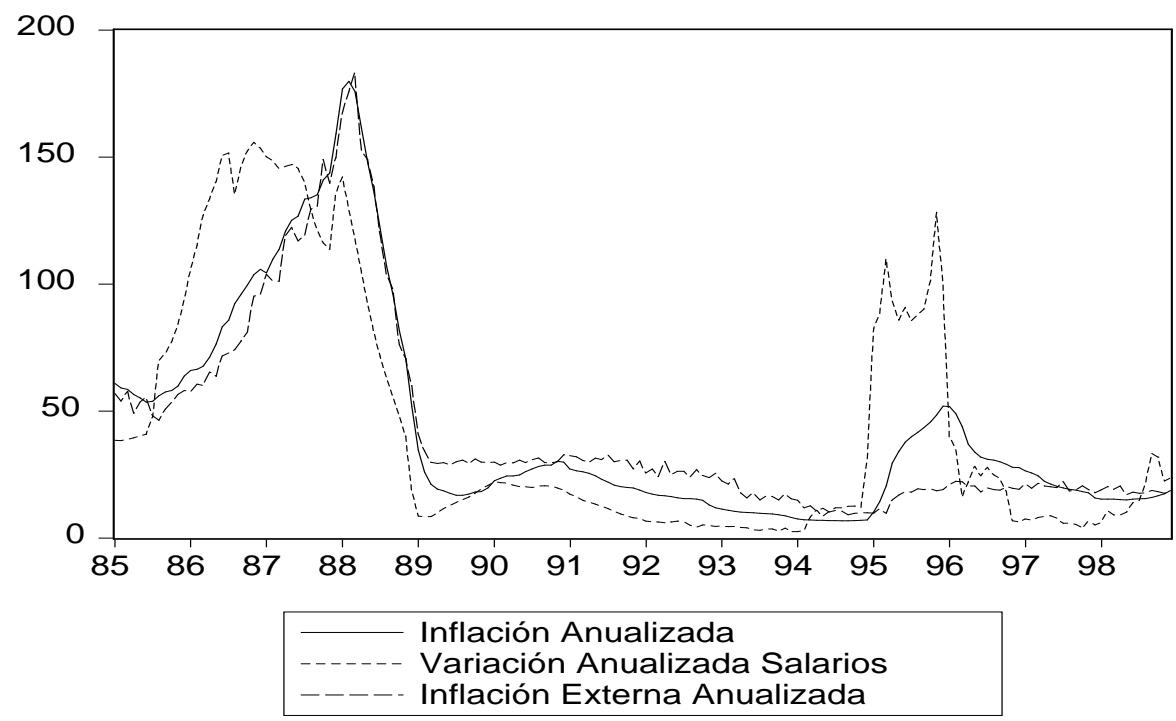

Gráfica 2: Relación entre inflación, tasa de crecimiento de los salarios y la inflación externa (porcentajes anualizados)

Otra variable que aparece en este estudio es el índice de la producción industrial, $Y$, sobre el cuál se calcula una medida de la brecha del producto. El método elegido para ello es el más simple, se utilizan los residuales (ydetrend) de una regresión de $y$ sobre una constante y una tendencia lineal. La regresión implica una tasa de crecimiento mensual promedio de $0.02 \%$ (ó $2.46 \%$ anual).

\section{Propiedades Estadísticas de las Series}

Las variables usadas en el documento tienen una propiedad estadística común. Esta es que la media y varianza de ellas no son constantes a través del tiempo. A esta característica se le denomina no-estacionareidad, e implica la necesidad de utilizar métodos estadísticos especiales. Las fuentes de esta propiedad son diversas. En este documento consideramos dos formas muy comunes. Una de ellas es la presencia de una tendencia determinística en la serie, como por ejemplo en la serie del índice de la produccción industrial, $y$. Otro es la existencia de una tendencia que cambia en cada período de manera aleatoria. A una serie con este último tipo de no-estacionareidad se le llama integrada de orden uno o $I(1)$. Si uno obtiene la primera diferencia de una serie que es $I(1)$ el resultado es una serie $I(0){ }^{6}$

\footnotetext{
${ }^{6}$ Todas las series que consideramos son a lo sumo $I(1)$ pero a menudo se encuentran series que son $I(2)$. En este caso el obtener la primera diferencia produce una serie que es $I(1)$.
} 
La demostración de que las series consideradas tienen alguna o ambas de estas formas de no estacionareidad se encuentra en el apéndice A. Allí se discuten las pruebas de raíces unitarias de Dickey Fuller Aumentada (ADF) y PhillipsPerron ( $\mathrm{PhP}$ ) para los logaritmos del índice general de precios al consumidor, el índice de precios controlados, el índice de salarios, los precios externos, índice de volumen de la producción industrial, el salario real y el tipo de cambio real.

Para los fines de este documento, los logaritmos del Indice Nacional de Precios al Consumidor $p$, el índice de precios no controlados $p n c$, el índice de precios administrados $p p$, del índice de salarios $w$ y de los precios externos epeu son variables $I(1)$, la desviación del logaritmo del índice de la producción industrial respecto a su tendencia, $y$, es $I(0)$ y tanto el logaritmo del salario real, $r w=(w-p)$, como el del tipo de cambio real, rer $=($ epeu $-p)$ son $\mathrm{I}(0)$. Estas características justifican el enfoque econométrico empleado en las siguientes secciones y el lector interesado puede acudir al apéndice referido, en donde se presentan los detalles del estudio estadístico .

\section{Relaciones de Largo Plazo}

\subsection{Marco Conceptual}

El marco conceptual utilizado es el de un modelo de inflación de costos unitarios similar al desarrollado por De Brower y Ericsson (1998) para el caso de Australia. En este modelo el nivel de precios es una función Cobb-Douglas de los salarios y los precios externos:

$$
P=\mu W^{\gamma_{w}} E P E U^{\gamma_{e}}
$$

donde $\gamma_{w}$ y $\gamma_{e}$ son las respectivas elasticidades de largo plazo (que bajo el supuesto de homogeneidad lineal deberían sumar 1) y $\mu$ es un coeficiente de margen sobre costos (mark up). Es útil escribir la ecuación anterior en logaritmos:

$$
p_{t}=\gamma_{w} w_{t}+\gamma_{e} \text { epeu }_{t}+\ln (\mu)
$$

Imponiendo la restricción de homogeneidad lineal la ecuación anterior se puede expresar de la siguiente manera:

$$
\gamma_{w}(w-p)+\gamma_{e}(\text { epeu }-p)=-\ln (\mu)
$$

Esto es, a partir de la ecuación de precios el margen sobre costos se puede reexpresar como una combinación lineal del logaritmo del salario real, $w-p$, y del logaritmo del tipo de cambio real, epeu $-p$, donde las ponderaciones son las respectivas elasticidades de largo plazo. De lo anterior se desprende que el modelo tiene como casos particulares el de la determinación del nivel de precios a través de la teoría de la paridad del poder de compra cuando $\gamma_{w}=0$ y el de 
la teoría de la determinación de precios a partir de los costos laborales cuando $\gamma_{e}=0 .^{7}$

Aunque el álgebra anterior se desarrolla únicamente en términos del logaritmo del índice nacional de precios al consumidor, $p$, el mismo modelo es aplicado al de precios no controlados, $p n c$, y puede utilizarse también con otros índices de precios de interés tales como los de bienes comerciables y no comerciables. Más adelante se introducen otras variables que posiblemente afectan la dinámica de corto plazo de la inflación (tales como la brecha de producto y los precios administrados).

\subsection{Estimación de la Relación de Largo Plazo}

Debido a la naturaleza de las series involucradas (esto es, la propiedad de no estacionareidad a la que nos referimos en la sección 3), el marco natural de análisis es el de cointegración. Antes de ir a los detalles de la estimación y pruebas de diagnóstico, puede ser útil discutir el concepto.

La noción de cointegración más difundida fue originalmente propuesta por Granger (1981). Usualmente es conocida como cointegración en el sentido de "Engle-Granger." Esta consiste en la existencia de un vector (o vectores) esta$b l e(\mathrm{~s})$ de coeficientes que producen una variable $I(0)$ a partir de variables $I(1) .^{8}$

Dichos vectores se intepretan como relaciones de equilibrio de largo plazo entre un conjunto de variables y a menudo es posible identificarlos con resultados derivados de la teoría económica. Esto resulta mucho más fácil cuando existe un solo vector de cointegración, como en el presente trabajo, donde dicho vector de coeficientes está relacionado con los parámetros de la ecuación (1).

Hay varios métodos para obtener los vectores de cointegración (si éstos existen). Estos caen dentro de dos categorías: a) métodos uniecuacionales y; b) métodos de sistemas. En este trabajo se utiliza el método más comúnmente empleado (el procedimiento de sistema de Johansen ( $\mathrm{JOH})$ ).

En esta sección se sintetizan los principales hallazgos. Los resultados completos del análisis de cointegración se reportan en los cuadros 3 y 4 del apéndice B. Se aplicó el método tanto al logaritmo del índice de precios al consumidor $p$ como al del índice de precios no controlados pnc. En ambos casos se comenzó y terminó con un sistema de doce rezagos para el que no fue posible simplificar a un número menor de ellos debido a que el doceavo rezago de la ecuación para la tasa de cambio de salarios, $\Delta w$, es fuertemente significativo.

Lo primero que conviene destacar es que en ambos casos se encontró un único vector de cointegración. Estos vectores normalizados son $\left(\beta_{p}, \beta_{w}, \beta_{e}\right)=$ $(1.00,-0.36,-0.63)$ у $\left(\beta_{p n c}, \beta_{w}, \beta_{e}\right)=(1.00,-0.45,-0.56)$. Estos resultados están relacionados con el modelo de la sección 4.1 haciendo que $\gamma_{w}=-\beta_{w}=$ 0.36 y $\gamma_{e}=-\beta_{e}=0.63$, es decir los componentes del vector de cointegración

\footnotetext{
${ }^{7}$ Aunque en este caso es común ajustar los salarios por el crecimiento de la productividad (ver por ejemplo Mehra (1994)).

${ }^{8}$ Desde luego que la noción se extiende de modo directo a órdenes de integración mayores, por ejemplo, variables I(2) pueden cointergrarse para producir variables que son I(1) o incluso $\mathrm{I}(0)$.
} 
debidamente normalizados se interpretan como los parámetros de la ecuación (1). Se observa que los vectores son ligeramente diferentes para el comportamiento del Indice Nacional de Precios al Consumidor y el índice de precios no controlados aunque ambos cumplen la propiedad de homogeneidad lineal

Se encontró que es indispensable que el índice de precios, el de salarios y el de precios externos se incluyan en la relación de equilibrio de largo plazo (En el apéndice B se presentan las pruebas de exclusión de estas variables). Las pruebas de exogeneidad débil indican que la única variable que es afectada por las desviaciones en el equilibrio de largo plazo es el índice de precios. Esto es importante para justificar el modelo uniecuacional de inflación que se presenta más adelante. ${ }^{9}$

Las pruebas de exogeneidad fuerte indican que la única variable con esta propiedad es el nivel de precios externos, epeu. Este resultado es muy importante pues indica que el tipo de cambio no puede ser pronosticado a partir de información pasada de precios y salarios mientras que pronósticos de estas variables si pueden ser mejorados con información proveniente del tipo de cambio. Se notará que estas pruebas de exogeneidad fuerte están muy asociadas a las pruebas de causalidad de Granger. ${ }^{10}$ Este resultado justifica la omisión de un modelo explícito para el tipo de cambio en los ejercicios de simulación de la sección 6 .

Es posible realizar un análisis similar al anterior con otros índices desagregados. Por ejemplo, es interesante evaluar el impacto de largo plazo de los costos laborales y el tipo de cambio sobre los bienes comerciables y no comerciables. Encontramos que para un índice de precios de bienes comerciables, la elasticidad con respecto a los salarios es considerablemente más baja que para el índice de precios de bienes no comerciables $(0.15$ contra 0.69$)$. Por contraste, la elasticidad con respecto a los precios externos es 0.81 y 0.32 , respectivamente. Estas magnitudes son consistentes con lo que uno esperaría: los salarios son mucho más importantes en la determinación de los precios de los bienes no comerciables que en el caso de los comerciables.

Un problema interesante, es la posibilidad de que la relación de largo plazo haya cambiado en vista de los importantes eventos ocurridos en México durante el período de muestra: el comienzo de la crisis de la deuda, la aparición de inflación acelerada y varios programas de ajuste macroeconómico, la apertura comercial y firma del tratado de libre comercio y la adopción de varios regímenes de tipo de cambio, entre otros.

Estudios preliminares demuestran que de haber ocurrido un cambio en la relación de largo plazo analizada, éste ocurrió a partir de la adopción del régimen de tipo de cambio flexible en Diciembre de 1994. Más adelante se examina el comportamiento de corto plazo de la inflación donde se muestra que éste, por el contrario, no parece haber sido afectado por los eventos mencionados anteriormente y ni siquiera por el probable cambio en la relación de largo plazo.

\footnotetext{
${ }^{9}$ Ver Johansen (1995), cap. 8.

${ }^{10}$ De hecho, exogeneidad débil más no causalidad en el sentido de Granger es equivalente a exogeneidad fuerte.
} 
Debido a que existe una multitud de métodos de estimación $\mathrm{y}$, dentro de estos, distintas especificaciones, es posible que un conjunto diferente de valores sea obtenido. Por ejemplo, uno de los métodos alternativos utilizados (el método FM-OLS) produce el vector $(1,-0.51,-0.49)$, el cual otorga similar peso a los salarios y a los precios externos. ${ }^{11}$

\subsection{Ajuste del Modelo de Largo Plazo}

El primer panel de la gráfica 3 muestra la trayectoria del logaritmo del Indice Nacional de Precios al Consumidor $(p)$ junto con la del siguiente estimado: ${ }^{12}$

$$
\hat{p}^{L P}=-6.25+0.36 w+0.63 \text { epeu }
$$

mientras que en el segundo panel se muestran las diferencias entre el nivel observado del índice nacional de precios al consumidor y el estimado $\hat{p}^{L P}$.
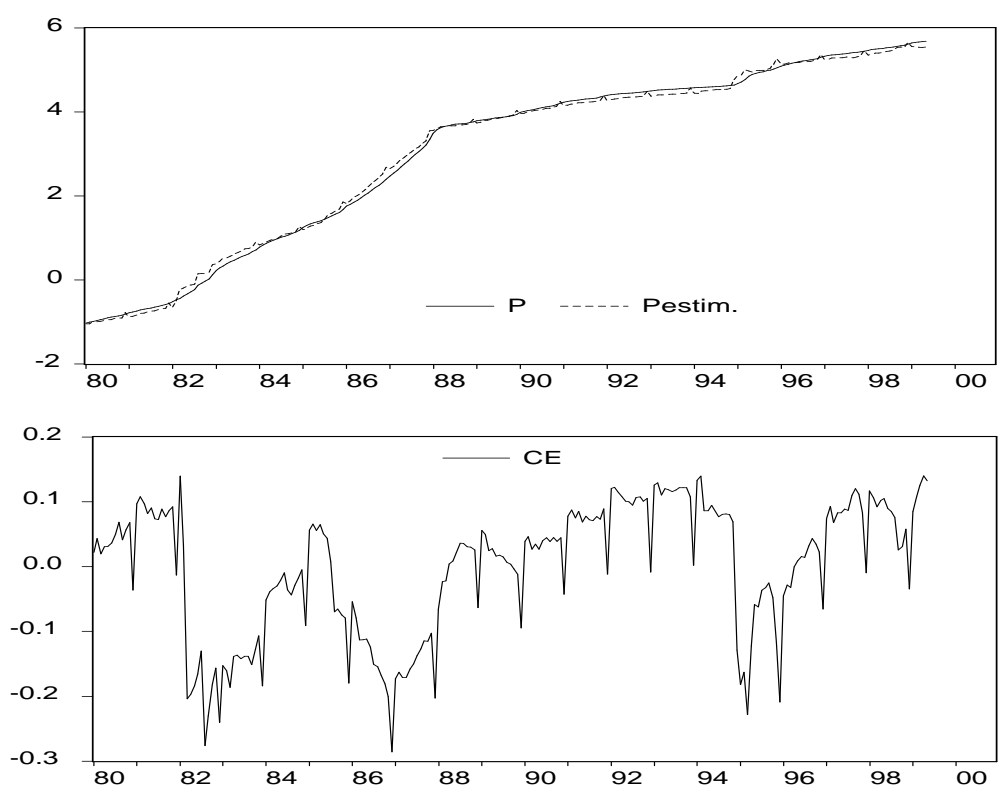

Gráfica 3: El Logaritmo del INPC $(p)$ y la "Solución de Largo Plazo" $\left(\hat{p}^{L P}\right)$.

\footnotetext{
${ }^{11}$ En un modelo en diferencias, Pérez-López (1996) encuentra resultados similares.

${ }^{12}$ Para representar la relación de equilibrio de largo plazo, elegimos los coeficientes obtenidos en el vector de corrección de error para el Indice Nacional de Precios al Consumidor por el método de Johansen. Este pareció ser el más robusto a distintas especificaciones y permitía un mayor ajuste del modelo de corto plazo que se presenta en la siguiente sección.
} 
Un estimado como $\hat{p}^{L P}$ es llamado a veces "la solución de largo plazo". Se forma con los coeficientes del vector de cointegración normalizado y los valores de las variables respectivas (en este caso $w$ y epeu). El ajuste parece bueno. Sin embargo, nótense los picos estacionales de $\hat{p}^{L P}$. Esto refleja el fuerte efecto estacional para el mes de diciembre en la serie utilizada para representar salarios. Por esta razón, el estimado de inflación mensual $\Delta \hat{p}_{t}^{L P}=\hat{p}_{t}^{L P}-\hat{p}_{t-1}^{L P}$ está condenado a comportarse pobremente en el mes de diciembre. ${ }^{13}$

Además, el hecho de que hasta este punto se ignoren factores que probablemente afecten la dinámica inflacionaria en el corto plazo hace necesaria la obtención de un modelo de inflación que incorpore todos esos elementos y al mismo tiempo sea consistente con "la solución de largo plazo". El procedimiento natural es la construcción de un modelo de corrección de error (lo que se lleva a cabo en la sección 5 ).

El segundo panel representa la diferencia entre $p$ y su estimado $\hat{p}^{L P}$, al cual se denomina término de corrección de error, $C E$ :

$$
C E_{t}=p_{t}-\hat{p}_{t}^{L P}
$$

Este término tiene un papel muy importante en la construcción de un modelo de inflación. Cuando es negativo, implica que la combinación ponderada de salarios y tipo de cambio excede el nivel de precios consistente con dicha combinación (es decir, $p<\hat{p}_{t}^{L P}$ ) por lo cual habrá presiones inflacionarias para restablecer el equilibrio. Cuando es positivo hay presiones deflacionarias. Por esta razón, un modelo de inflación que no lo incorpore entre sus variables explicatorias probablemente estará mal especificado.

Un resultado adicional es que aunque $\Delta \hat{p}_{t}^{L P}$ es un mal estimado de la inflación mensual, el estimado de la inflación anualizada $\Delta_{12} \hat{p}_{t}^{L P}=\hat{p}_{t}^{L P}-\hat{p}_{t-12}^{L P}$ puede ser más razonable. La razón es que con la diferencia anual los factores estacionales se eliminan y el efecto de los factores de corto plazo se atenúa.

\section{El Modelo de Corto Plazo para la Inflación}

En esta sección se lleva a cabo la segunda etapa del proceso de modelaje. El objetivo es el de representar la dinámica de ajuste de las variables de interés. Esto requiere derivar un modelo de corrección de error para al menos una de las variables, en este caso el índice de precios. Dados los resultados obtenidos antes, donde se demostró que $p$ y $p n c$ son variables débilmente endógenas ${ }^{14}$ en sus respectivos sistemas, es posible desarrollar modelos de corrección de error de una sola ecuación para precios $(p)$ y precios no controlados $(p n c)$ condicionando en las otras variables, lo que se hace a continuación.

\footnotetext{
${ }^{13}$ Ver Hatanaka, M. (1996),Hendry, D.F. (1995) y Johansen, S. (1995)

${ }^{14}$ Los salarios en el sistema de los precios no controlados pnc también aparecían como variable débilmente endógena. Tal resultado, sin embargo no se sostiene al tratar de estimar un modelo de corrección de error para esta variable. El coeficiente correspondiente al término de corrección de error es no significativo a niveles del $40 \%$. Por esta razón se optó por tomar a pnc como la única variable débilmente endógena en el sistema correspondiente.
} 


\subsection{Derivación del Modelo de Corrección de Error para la Inflación}

Existen varias alternativas para derivar el modelo de corrección de error. El punto de partida elegido para obtener dicho modelo fue el de plantear ecuaciones generales para la inflación general, $d p=\Delta p$, y la del índice de precios de bienes controlados, $d p n c=\Delta p n c$, de la siguiente forma:

$$
\begin{aligned}
\mathrm{dp}_{\mathrm{t}}=\mu_{\mathrm{p}}+ & \alpha \mathrm{CE}_{\mathrm{t}-\mathbf{1}}+\sum_{\mathbf{i}=\mathbf{0}}^{12} \phi_{1, \mathbf{i}} \mathrm{d} \mathbf{w}_{\mathrm{t}-\mathbf{i}}+\sum_{\mathbf{i}=0}^{12} \phi_{2, \mathbf{i}} \operatorname{depeu}_{\mathrm{t}-\mathbf{i}}+\sum_{\mathbf{i}=\mathbf{0}}^{12} \phi_{3, \mathbf{i}} \mathrm{dpp}_{\mathrm{t}-\mathbf{i}} \\
& +\sum_{\mathbf{i}=\mathbf{0}}^{12} \phi_{4, \mathbf{i}} \operatorname{ydetrend}_{\mathrm{t}-\mathbf{i}}+\sum_{\mathbf{i}=\mathbf{1}}^{12} \xi_{\mathbf{i}} \mathrm{dp}_{\mathrm{t}-\mathbf{i}}+\phi \mathbf{X}_{\mathrm{t}}+\mathbf{u}_{\mathrm{t}}
\end{aligned}
$$

Lo primero que uno debe observar es la presencia del término de corrección de error, $C E_{t}$, (descrito en la sección 4.3). Este término representa las presiones inflacionarias (o deflacionarias) que provienen de algún desequilibrio en la relación de largo plazo y es lo que da a la ecuación el carácter de modelo de corrección de error. Además se incluyen rezagos y valores contemporáneos de la inflación salarial $d w=\Delta w$ y la externa depeu $=\Delta$ epeu.

La matriz de variables exógenas ahora incluye otras más que pueden afectar a la inflación en el corto plazo. Entre las variables exógenas adicionales se incluyen valores contemporáneos y rezagados de la inflación de precios públicos, ${ }^{15} d p p=$ $\Delta p p$ y de la brecha de producto, ydetrend. Una ecuación similar se construyó para la inflación de precios no controlados, dpnc.

A partir de estas ecuaciones generales se eliminaron los términos no significativos para llegar a los modelos de corto plazo. Se evitó el uso de variables dicótomas (excepto de las estacionales) para reducir el riesgo de sobreparametrización.

\subsection{Interpretación Económica de los Resultados}

El cuadro 1 muestra los resultados de los modelos que sobrevivieron al proceso de depuración general a específico, con la excepción de la constante y las variables estacionales. Para ambas ecuaciones la estructura final fue la misma. En primer lugar, hay que destacar que en ninguna de las ecuaciones aparecen rezagos de orden mayor que el primero. Luego, se observa un valor moderado del coeficiente de autorregresión de primer orden que va de 0.44 para el modelo de la inflación general, $d p$, a 0.36 para el modelo de la inflación de precios no controlados, dpnc. Este coeficiente indica que por cada punto porcentual de la inflación del período anterior, alrededor de $0.4 \%$ se reflejará en la inflación presente.

Esto contrasta con el modelo autorregresivo más sencillo donde tal coeficiente puede llegar a ser tan alto como 0.90, dando la impresión de que la inflación

\footnotetext{
${ }^{15}$ Los cuales han afectado a la inflación del resto de los bienes de diversas maneras (Para una discusión profunda de estos mecanismos véase Gamboa (1997))
} 
es un proceso con raíz unitaria (o puramente inercial). Este resultado implica que después de considerar las variables relevantes, la parte inercial (aquella parte de la inflación presente explicada únicamente por su historia) se reduce sustancialmente. ${ }^{16}$

Cuadro 1: Modelos de Inflación para $p$ y
\begin{tabular}{|l|c|c|}
\hline VARIABLES & $d p_{t}$ & $d p n c_{t}$ \\
\hline \hline$d p n c_{t-1}$ & $\cdot$ & 0.442 \\
& $\cdot$ & $(0.052)$ \\
\hline CEpnc $t-1$ & $\cdot$ & -0.053 \\
& $\cdot$ & $(0.009)$ \\
\hline$d p_{t-1}$ & 0.364 & $\cdot$ \\
& $(0.033)$ &. \\
\hline CE $p_{t-1}$ & -0.048 & $\cdot$ \\
& $(0.009)$ &. \\
\hline$d w_{t}$ & 0.081 & 0.089 \\
& $(0.019)$ & $(0.022)$ \\
\hline depeut & 0.044 & 0.042 \\
& $(0.010)$ & $(0.011)$ \\
\hline$d p p_{t}$ & 0.366 & 0.112 \\
& $(0.024)$ & $(0.025)$ \\
\hline ydetrend $u_{t-1}$ & $\cdot$ & 0.022 \\
&. & $(0.011)$ \\
\hline \hline T (85:02 - 98:12) & 167.00 & 166.00 \\
$R^{2}$ & 0.97 & 0.95 \\
$R^{2}$ Ajustada & 0.96 & 0.94 \\
E.E. Regresión & 0.004 & 0.01 \\
Coef. Asimetría & 0.27 & 0.17 \\
Curtosis & 7.80 & 3.89 \\
Est. Jarque-Bera & $162.26^{* *}$ & 6.32 \\
LM (12) AR & 0.891 & 0.98 \\
LM (12) ARCH & $2.24^{*}$ & 1.39 \\
Prueba de White & $7.28^{* *}$ & $3.21^{* *}$ \\
Prueba RESET $(1)$ & $22.12^{* *}$ & $9.82^{* *}$ \\
\hline
\end{tabular}

El coeficiente para el término de corrección de error rezagado (conocido como velocidad de ajuste) tiene signo negativo, lo cual es requerido para que la dinámica de la ecuación sea estable: partiendo de una situación de equilibrio, un valor negativo del término de corrección de error, causado por un aumento en salarios o precios externos, implica una presión inflacionaria que se corregirá gradualmente en períodos subsiguientes con el aumento del nivel de precios doméstico.

Mientras mayor sea el valor absoluto del coeficiente, más rapido será el ajuste. El valor absoluto de la velocidad de ajuste para ambas ecuaciones es muy similar (0.048 para precios y 0.052 para precios no controlados). Sin embargo,

\footnotetext{
${ }^{16}$ Compárese este resultado con el reportado por Dureval (1998) para el caso de Brazil en el período 1968-1985, conocido como de inflación crónica y que precedió al de hiperinflación. Este autor estimó un grado de inflación inercial de 0.41. En un modelo similar para Australia, de De Brower y Ericsson (1998) encuentran un valor de cero.
} 
el que el coeficiente del término autorregresivo sea menor para la inflación del Indice Nacional de Precios al Consumidor implica que los ajustes tomarán más tiempo que en el caso de la inflación de precios no controlados. Esto es explicable porque los precios de bienes controlados están incluídos en el primero de estos índices y éstos pueden tardar más tiempo en responder a los cambios en salarios y precios externos.

Es importante enfatizar que el efecto de las desviaciones en la ecuación de precios sobre la inflación tiene lugar hasta el período siguiente. Esto difiere de los efectos de corto plazo que pueden afectar a la inflación de manera contemporánea (además de o en lugar de manera retardada). Se observa que la tasa de crecimiento de los salarios (un efecto de corto plazo) sólo impacta a la inflación de manera contemporánea y con una magnitud muy similar en ambas ecuaciones. El efecto es tal que si los salarios crecen $1.0 \%$ en el período actual, la inflación del INPC se incrementa en $0.08 \%$ mientras que para la inflación de no controlados se incrementa en $0.09 \%$.

Similarmente, si la inflación importada aumenta en 1.0\% la inflación del INPC y la de bienes no controlados se incrementará en alrededor de $0.04 \%$ de manera inmediata. No hay que perder de vista que éstos son efectos de corto plazo (o inmediatos) de ajustes salariales o de precios externos o fluctuaciones del tipo de cambio. El efecto total se irá acumulando a lo largo del tiempo a través de la influencia proveniente del término de corrección de error.

La inflación contemporánea de bienes controlados se incluye para capturar los efectos indirectos que ajustes en estos precios tienen sobre el resto de los precios en la economía. El coeficiente para esta variable es el que más difiere en ambas ecuaciones (0.37 para el la inflación del Indice Nacional de Precios al Consumidor, $d p$, y 0.11 para la del índice de precios controlados, $d p n c$ ) y la razón es evidente: los precios de bienes controlados están incluidos en el índice de precios $(p)$ así que la inflación de precios públicos $(d p p)$ en realidad se encuentra en ambos lados de la ecuación. Esto sin duda exagera el efecto de $d p p$ sobre los otros precios. El omitir esta variable de la ecuación ocasiona un deterioro en el desempeño estadístico de algunos aspectos del modelo (la distribución del término de error es mucho más asimétrica) pero lo mejora en otros (elimina todo vestigio de efectos $\mathrm{ARCH}$ ).

La última variable que sobrevivió en la ecuación para la inflación de precios no controlados (pero no para la inflación del INPC) fue la brecha de producto. Se probaron dos medidas para ésta, la cual es una variable, fundamental en los modelos de inflación basados en la curva de Phillips. Primero, se utilizó la más simple que consiste en utilizar los residuales de una regresión del logaritmo de la producción industrial contra una constante y una tendencia. Segundo, se calculó la diferencia de la serie del logaritmo de la producción industrial y una tendencia dada por el filtro de Hodrick-Prescott. ${ }^{17}$ Los resultados no fueron muy diferentes y sólo se reporta el caso más sencillo. Por cada punto porcentual que la actividad económica se desvíe de su tendencia la inflación de precios no controlados aumentará en 0.04 .

\footnotetext{
${ }^{17}$ Ver Hodrick y Prescott (1997).
} 
Se probaron otras variables que podrían ayudar a explicar la inflación en México tales como la tasa de cambio de varios agregados monetarios, de salarios, de la productividad y del salario mínimo. En el caso de los agregados monetarios sólo el coeficiente de la tasa de cambio de M1 resultó marginalmente significativo (pero con un signo negativo,lo que no tiene mucho sentido económico).

También se probó el término de corrección de error de una demanda de dinero. ${ }^{18}$ Este término tiene la interpretación de medida de desequilibrio en el mercado monetario. Introduciendo este término, la ecuación a estimar es similar a la del modelo para la inflación en Dinamarca desarrollado por Juselius (1992) ${ }^{19} \mathrm{El}$ coeficiente para este término también resultó no significativo.

El resultado anterior no es equivalente a afirmar que los agregados monetarios no tienen influencia alguna en la dinámica inflacionaria. La manera en la que éste se interpreta es que el dinero afecta a la inflación de manera indirecta, a través de sus efectos en la determinación del tipo de cambio, por ejemplo. En este sentido estas ecuaciones ayudan a entender cuál es el mecanismo de transmisión de la política monetaria en México. Finalmente, el efecto de los cambios en la productividad y el salario mínimo fue insignificante en todas las especificaciones.

\subsection{Pruebas de Diagnóstico}

Las pruebas de diagnóstico resultan ser satisfactorias. Sólo la ecuación para la inflación del Indice Nacional de Precios al Consumidor, $d p$, muestra problemas de asimetría y exceso de curtosis importantes que provocan que la hipótesis de normalidad de los residuales se rechace. Para la ecuación de $d p n c$ el rechazo de la hipótesis de normalidad es marginal.

Los estadísticos $L M$ muestran ausencia de correlación serial y solamente en la ecuación para $d p$ se encuentra evidencia marginal de efectos $\mathrm{ARCH} .{ }^{20}$ Esto lleva a creer que estos efectos no son un problema en las ecuaciones analizadas. Este resultado es importante en sí mismo ya que representa una objeción a la extendida práctica de modelar la varianza de la inflación en México y otros países $^{21}$ (a menudo interpretada como una medida de la incertidumbre inflacionaria) como un proceso ARCH.

\footnotetext{
${ }^{18} \mathrm{Se}$ utilizó el término de corrección de error de la ecuación de demanda de dinero que para México estimaron Khamis y Leone (1999). Estos autores arguyen que dicha demanda es estable aunque sólo prueban la estabilidad del modelo de corto plazo.

${ }^{19} \mathrm{La}$ ecuación de inflación que estos autores estiman tiene tres términos de corrección de error estimados de manera separada. Estos términos son el salario real, el tipo de cambio real y las desviaciones de largo plazo de una demanda de dinero. La variación que se adopta para la ecuación estimada en este documento es que los datos imponen el mismo coeficiente para el salario real y el tipo de cambio real además de un coeficiente cero para el error de equilibrio en el mercado monetario.

${ }^{20}$ Autoregressive Conditional Heteroskedasticity. Esta propiedad estadística se halla frecuentemente en modelos de series financieras pero en este contexto es simplemente otra prueba de especificación más: si el modelo está bien especificado en una forma lineal entonces estos defectos no deberían de presentarse.

${ }^{21}$ Ver por ejemplo Magendzo (1998), Crawford y Kasumovich (1996) y Grier (1998)
} 
Es posible que lo que tales modelos capturan sea una mala especificación de la media condicional o alguna forma alternativa de no linealidad. La ecuación para $d p n c$, sin embargo, no está libre de problemas de heterocedasticidad pues el estadístico de White rechaza la hipótesis de varianza constante. ${ }^{22}$ Por esta razón, todos los errores estándar están corregidos por el método de White para ser robustos a dicho problema.

Es probable que este mismo problema sea responsable de la falla de los modelos para pasar la prueba RESET, la cual captura problemas de especificación y/o no linealidad en las series. Esta impresión se basa en que el único término que es significativo en la regresión auxiliar es el correspondiente a los residuales al cuadrado (el de residuales al cubo, que captura asimetría, no lo es). En el apéndice $\mathrm{C}$ se lleva cabo el análisis de la constancia del modelo.

La bondad de ajuste, medida por el coeficiente de determinación múltiple ajustado, es muy alta (de 0.94 a 0.96 ). Esto se puede observar en la gráfica 4 donde se representan las series observadas y ajustadas de inflación para el modelo dpnc (la del otro modelo es muy similar).

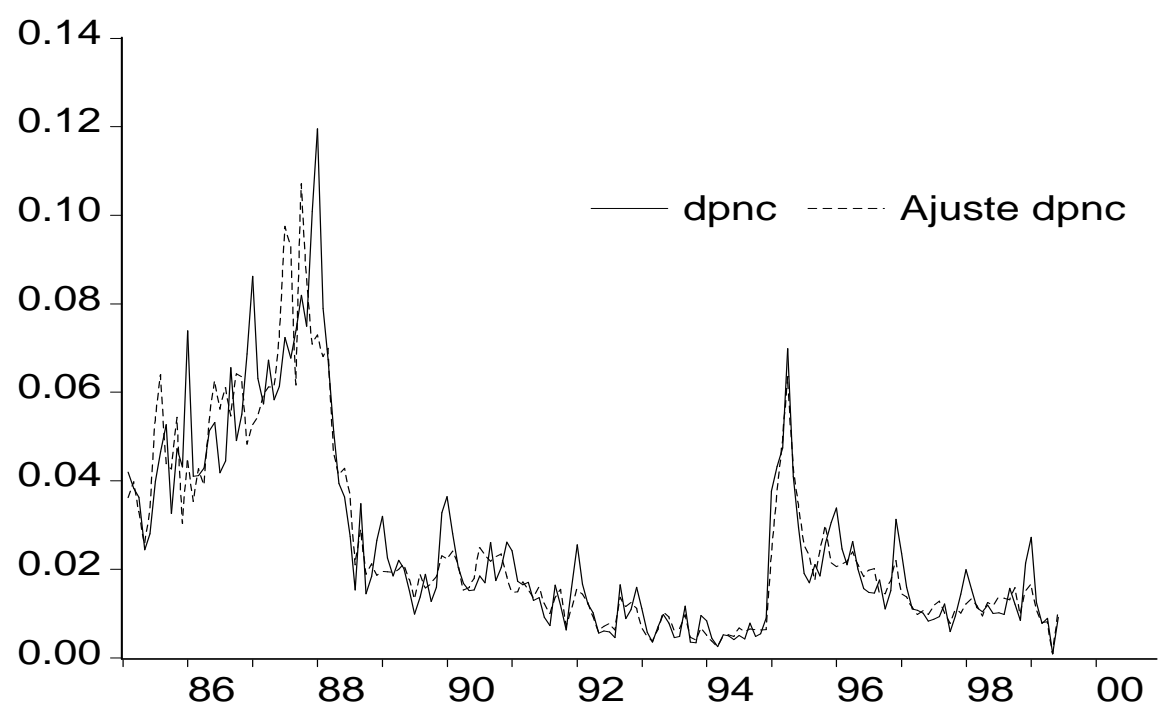

Gráfica 4: Ajuste del modelo de inflación.

\footnotetext{
${ }^{22} \mathrm{La}$ presencia de estos efectos en modelos lineales captura problemas de especificación pero no se descarta que también reflejen alguna forma de no linealidad en la serie
} 


\section{Implicaciones Dinámicas del Modelo}

El modelo desarrollado en las secciones anteriores tiene propiedades dinámicas interesantes que serán exploradas en esta sección. Para ello se muestran las respuestas de la inflación ante cambios en sus determinantes principales. Se utilizará el caso de la inflación de precios no controlados, dpnc, para hacer algunos de los ejercicios comparables con los resultados obtenidos para el caso de Australia. ${ }^{23}$

Para completar el sistema, se estimaron ecuaciones para los salarios y la brecha de producto. El modelo de salarios es presentado y analizado con cierto detalle en el apéndice D. Para la brecha de producto se estimó un modelo AR(5) con algunas variables exógenas adicionales. El modelo para precios externos se formó de dos partes. Un simple modelo autorregresivo para los precios de Estados Unidos y el tipo de cambio nominal que se supone exógeno al modelo. Esto es justificable en base a las pruebas de exogeneidad fuerte de la sección 4.2 $\mathrm{y}$ del apéndice $\mathrm{B}$.

Los precios públicos son considerados también una variable exógena en el corto plazo por lo cual, al menos que se diga lo contrario, se supone que permanecen constantes durante las simulaciones. Solamente en la sección 6.2 este supuesto y el del tipo de cambio son relajados. Las ecuaciones estimadas son las siguientes:

$$
\begin{aligned}
& p n c_{t}=0.36 w_{t}+0.63 \text { epeu }_{t}-6.25 \\
& d p n c_{t}=0.42 d p n c_{t-1}-0.06 C E_{t-1}+0.09 d w_{t} \\
& +0.04 \text { depe }_{t}+0.011 d p p_{t} \\
& +0.02 \text { ydetrend }_{-1} \\
& d w_{t}=-0.24 d w_{t-1}+0.03 d w_{t-3}+0.31 d w_{t-12} \\
& +0.65 d p n c_{t-1}+0.38 d p p_{t-1} \\
& +0.06 \text { ydetrend }_{t-1} \\
& \text { ydetrend }_{t}=0.38 \text { ydetrend }_{t-1}+0.48 \text { ydetrend }_{t-2} \\
& -0.36 \text { ydetrend }_{t-3}-0.15 \text { ydetrend } d_{t-4} \\
& -0.15 \text { ydetrend } d_{t-5}-0.13 d w_{t-2} \\
& -0.10 \text { depe }_{t-1}
\end{aligned}
$$

\footnotetext{
${ }^{23}$ Como pnc excluye los precios controlados, la comparación con el modelo de De Brower y Ericsson es más razonable debido a que estos dos autores usan un índice de precios de inflación subayacente. Los índices, aunque no iguales son más comparables que si se utilizara $p$.
} 


\subsection{La Dinámica de los Efectos de Salarios, Tipo de Cam- bio, Precios Públicos y Brecha de Producto sobre Pre- cios no Controlados}

Este ejercicio muestra la transmisión (pass-through) de ajustes salariales y cambiarios sobre el nivel de precios no controlados partiendo de un estado de cero inflación. La línea curva punteada de la gráfica 5 representa el efecto acumulado a través del tiempo de un ajuste salarial de $10 \%$ manteniendo invariantes al tipo de cambio, los precios públicos y la brecha de producto. La línea curva sólida de la misma gráfica representa el efecto acumulado de un ajuste del tipo de cambio de $10 \%$ manteniendo constantes a los precios públicos, los salarios y la brecha de producto. Las líneas horizontales hacia las que las curvas tienden indican los respectivos efectos de largo plazo.

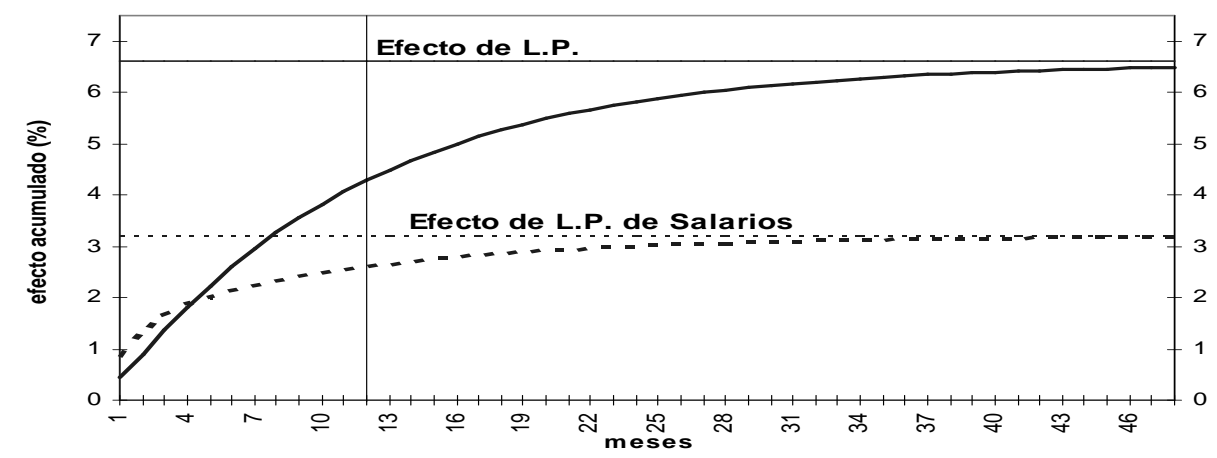

Gráfica 5: La Incorporación Gradual de Ajustes Salariales y Cambiarios.

Se observa que hasta el cuarto mes, el efecto de un aumento salarial del $10 \%$ es mayor que el de una depreciación equiparable del tipo de cambio. Debido a que la elasticidad de largo plazo es mayor para este último, el efecto de una depreciación eventualmente se vuelve más importante. La velocidad de ajuste del sistema a un movimiento salarial es mayor que para una fluctuación cambiaria. Esto se puede ver notando que la curva para salarios se aproxima más rápido a la recta que marca su efecto de largo plazo. Al término de un año (señalado con la línea vertical) $86 \%$ del impacto de largo plazo del ajuste salarial se ha materializado, mientras que después de un movimiento cambiario sólo el $83 \%$ de su efecto se transmite a precios en un lapso similar. 
Vale la pena enfatizar que en el sistema de ecuaciones presentado, un ajuste salarial no tiene efectos sobre el tipo de cambio pero que movimientos en éste si afectan a los salarios a través de los términos de inflación rezagada que aparecen en la ecuación 9. Esta propiedad no es arbitraria debido a que las pruebas estadísticas del apéndice $B$ muestran que el tipo de cambio es fuertemente exógeno con respecto al nivel de precios y al de salarios.

La gráfica 6 muestra la trayectoria del efecto acumulado sobre los precios no controlados y los salarios proveniente de un incremento de $10 \%$ en los precios públicos manteniendo constantes a el tipo de cambio y la brecha de producto. La curva punteada muestra el efecto de largo plazo sobre precios no controlados y la línea sólida muestra el impacto sobre salarios.

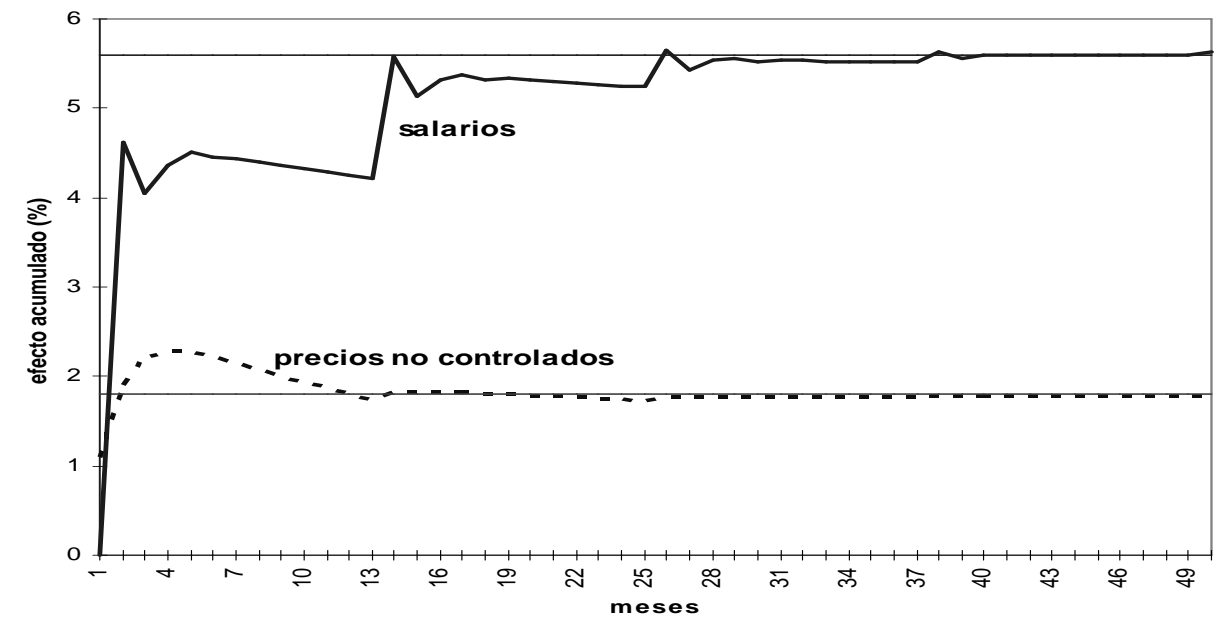

Gráfica 6: La Incorporación Gradual de Choques de Precios Públicos Sobre Precios no Controlados y Salarios.

El efecto de largo plazo de un ajuste de $10 \%$ en los precios públicos sobre los precios no controlados se estimó en $1.8 \%$, nivel hacia el cual la curva converge en la gráfica. Nótese que al comienzo hay un sobre-efecto que después desaparece y que la incorporación del choque es mucho más rápida que en el caso de los salarios y del tipo de cambio. Ambos fenómenos suceden por el fuerte efecto de corto plazo que los precios públicos tienen directamente sobre los precios no controlados (el coeficiente asociado a la inflación de precios públicos, $d p p$, en la ecuación (8), que es de 0.11). El efecto total sobre salarios se estimó en 5.6\%, nivel hacia el cual la curva de salarios converge rápidamente. 
Se realizó un ejercicio similar para analizar el efecto acumulado sobre precios no controlados y salarios proveniente de un choque de $10 \%$ a la brecha del producto manteniendo constantes al tipo de cambio y los precios públicos. Los resultados se presentan en la gráfica 7 . Nuevamente, la curva punteada muestra el efecto de largo plazo sobre los precios no controlados y la sólida sobre los salarios.

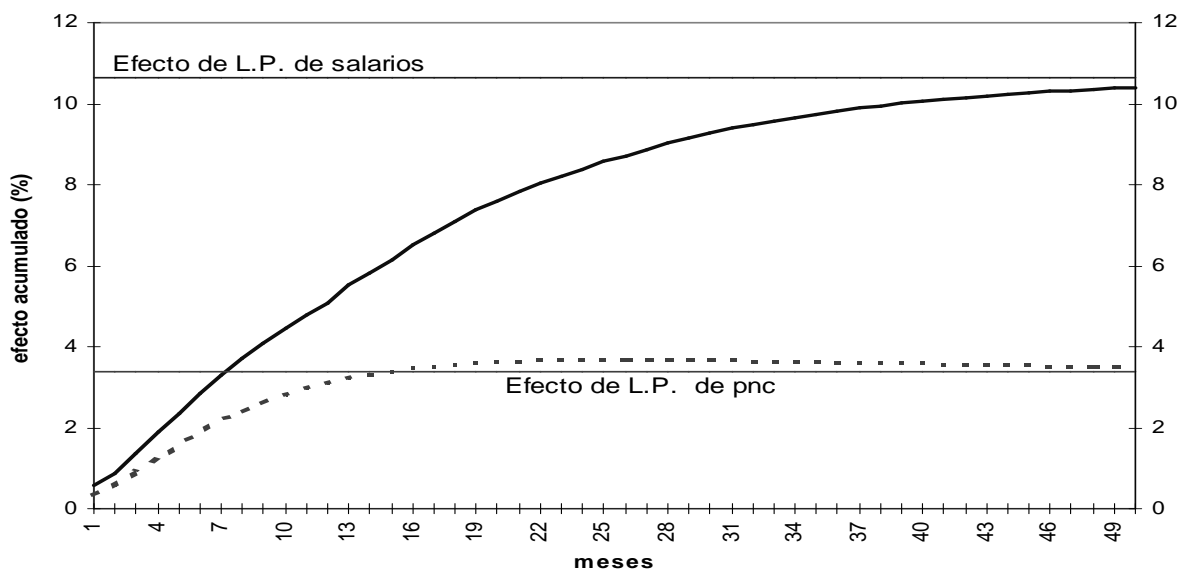

Gráfica 7: La Incorporación Gradual de Choques de la Brecha de Producto sobre Precios no Controlados y Salarios.

El efecto de largo plazo de un choque de $10 \%$ a la brecha de producto sobre los precios no controlados se estimó en $3.4 \%$ mientras que en los salarios el efecto fue de $10.62 \%$. Estos números son más altos que los correspondientes a una variación similar de precios públicos. Sin embargo, se debe tomar en cuenta que una brecha de producto de $10 \%$ es un evento muy improbable. Finalmente, se observa que la convergencia no es tan rápida como en el caso de un choque a los precios de bienes públicos. 


\subsection{Dinámica de la inflación a Ajustes Salariales con Res- puesta del Tipo de Cambio y Precios Públicos}

Todos los ejercicios previos han asumido que tanto los precios públicos como el tipo de cambio no son afectados por movimientos en las otras variables. En ciertas circunstancias este supuesto es extremo. En la sección 2 se discute cómo el comportamiento de los precios públicos ha variado a lo largo del tiempo. De acuerdo a esa discusión, el supuesto de que éstos se mantienen constantes durante el período de ajuste no parece realista.

Asimismo, es muy probable que movimientos inflacionarios deban ser compensados en el mediano plazo con ajustes del tipo de cambio nominal para mantener, por ejemplo, cierto nivel de tipo de cambio real, siempre y cuando no se presenten factores que alteren el nivel de equilibrio de esta variable. Para analizar el efecto de estos ajustes de precios públicos y tipo de cambio, se proponen las siguientes ecuaciones de comportamiento para estas variables.

$$
\begin{aligned}
d p p_{t} & =d p n c_{t-1} \\
d e_{t} & =0.5\left(e_{t-1}-p_{t-1}\right)
\end{aligned}
$$

La ecuación 11 dice que los precios públicos se rezagan con respecto a los precios no controlados a lo más un período. La ecuación 12 asume que el tipo de cambio nominal se deprecia como una proporción del desalineamiento del tipo de cambio real en relación a cierto nivel de "equilibrio" (que en este ejercicio se normaliza a cero y no cambia durante el período de simulación). La velocidad de ajuste para los precios públicos se asume mayor que la del tipo de cambio, lo que corresponde a lo observado durante el período de estudio aunque las magnitudes específicas se eligen únicamente por sencillez.

No está por demás enfatizar que ninguna de estas dos ecuaciones proviene de un proceso de estimación y ellas sólo representan mecanismos de ajuste razonables para los precios públicos y el tipo de cambio que aquí utilizamos para exponer las propiedades dinámicas del modelo.

La gráfica 8 muestra cuál es el efecto acumulado de un ajuste de $10 \%$ en salarios cuando el tipo de cambio nominal y los precios públicos se mueven de acuerdo a las ecuaciones 11 y 12 . La línea horizontal más elevada corresponde al nuevo efecto de largo plazo del ajuste salarial mientras que la horizontal más baja corresponde al efecto de largo plazo manteniendo todas las otras variables constantes. La línea curva representa el efecto acumulado del choque salarial sobre precios no controlados a lo largo del tiempo. 


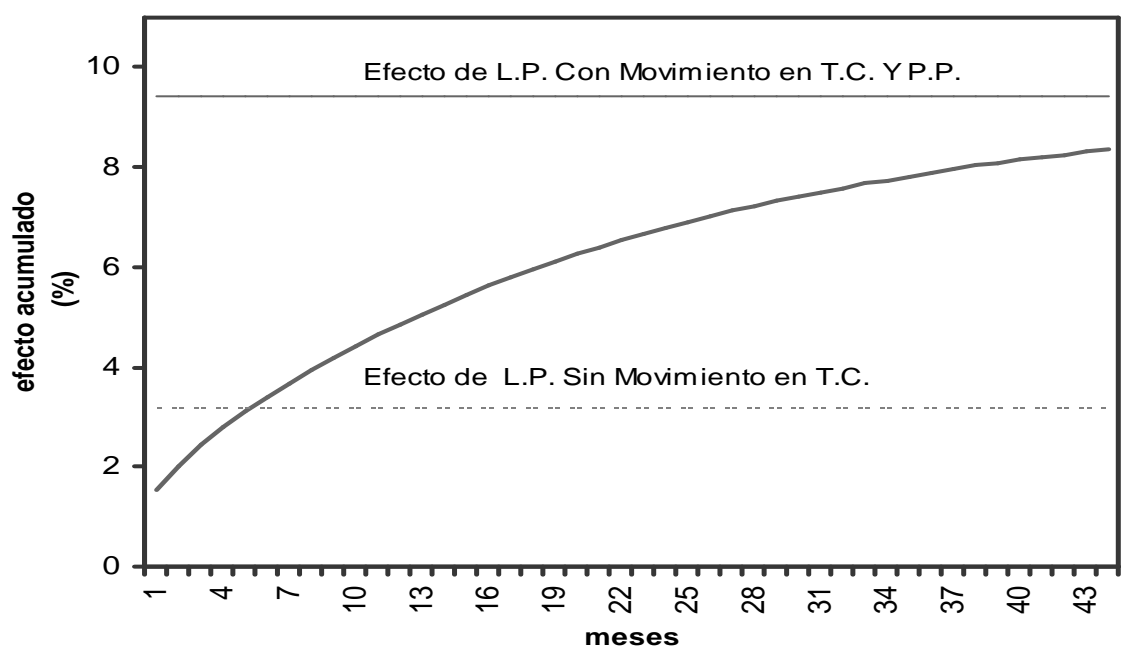

Gráfica 8: Efectos de un Ajuste Salarial con Respuesta del Tipo de Cambio Nominal y Precios Públicos.

Se puede observar que en este caso el efecto total original de $3.2 \%$ es alcanzado por la nueva curva en apenas cinco meses y que en un año el efecto acumulado es de 5.0\%. Para el segundo año los precios no controlados se han incrementado en $6.8 \%$ mientras que para el cuarto el porcentaje de incremento alcanza el $9 \%$. El tiempo en que el efecto total (9.6\%) se transfiere excede los cinco años. Este ejercicio muestra cómo una vez que los mecanismos de ajuste de precios públicos y tipo de cambio entran en acción, las ganancias de un incremento salarial se erosionan de modo rápido por el aumento de la inflación.

\subsection{Comparación con Australia}

La siguiente simulación (gráfica 9) compara el efecto inflacionario de un choque cambiario en México y Australia. Estos dos países tienen una elevada elasticidad de largo plazo para el tipo de cambio (0.63 y 0.44 , respectivamente). Esto es así por ser ambas economías pequeñas y abiertas. La diferencia entre las dos estriba en la velocidad con la que una depreciación se transmite al nivel general de precios.

Por sencillez y para facilitar la comparación, se supone que todas las otras variables permanecen constantes durante el período de simulación. La gráfica muestra para cada país el efecto acumulado sobre el nivel de precios causado por una depreciación de $10 \%$ en un horizonte de 1 a 12 trimestres.

En los primeros seis meses en México se ha transferido el $54 \%$ del efecto 


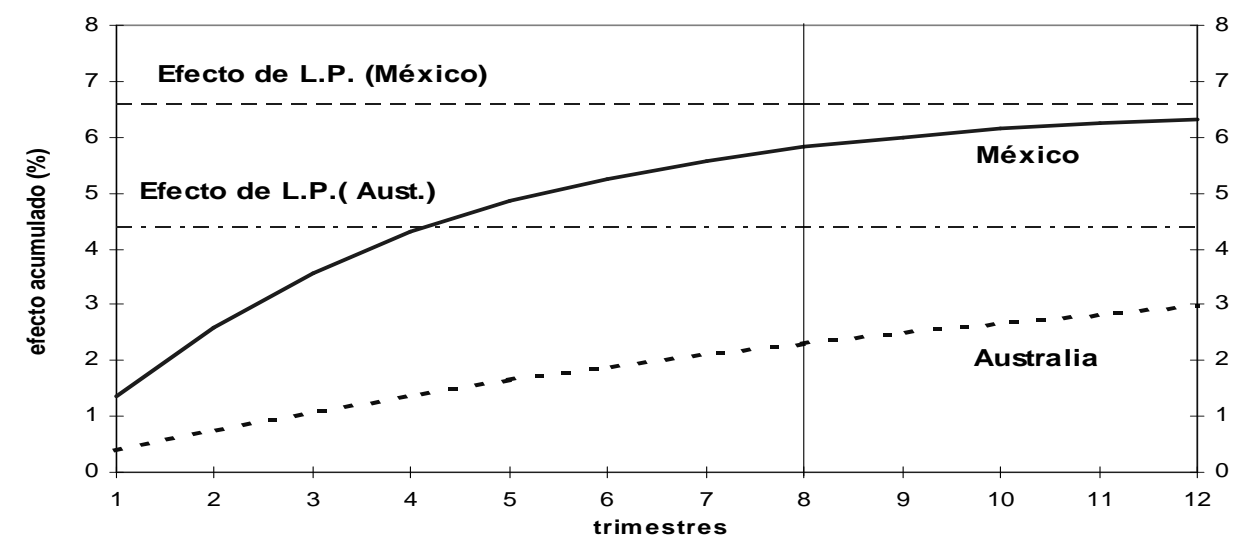

Gráfica 9: Comparación de la Incorporación Gradual de Choques al Tipo de Cambio en México y Australia.

total de la depreciación contra apenas el $17 \%$ en Australia. En doce meses, los números son $83 \%$ en México y $31 \%$ en Australia. Al cabo de dos años, solamente la mitad del efecto total se ha incorporado en Australia mientras que en México la transmisión se ha llevado a cabo en $92 \%$.

\section{Conclusiones}

En esta investigación se han derivado relaciones de largo plazo para dos índices de precios, el INPC y un índice de bienes no controlados, con sus determinantes principales, el nivel de salarios y los precios externos. Asimismo, se derivaron los correspondientes modelos de corrección de error para representar la dinámica de ajuste del corto plazo. Estos modelos son propuestos como modelos de inflación para el período 1985-1998.

Partiendo del hecho de que los logaritmos de los índices de precios, salarios y precios externos son variables no estacionarias $(I(1))$ se establece que estas variables están relacionadas a través de la existencia de un único vector de cointegración, que al ser normalizado se interpreta como una ecuación de precios de largo plazo. Dicho resultado, junto con el de la homogeneidad lineal, es sumamente robusto al período de muestra utilizado.

Se estima que la elasticidad de largo plazo del nivel de precios con respecto a los salarios para toda la muestra es igual a 0.36 mientras la correspondiente 
para los precios externos es 0.63 . Sin embargo, es posible que esta relación de largo plazo haya cambiado a partir de la adopción del régimen de flotación del tipo de cambio por parte de las autoridades mexicanas.

Los modelos de corto plazo para la inflación se derivan del proceso de corrección de error implícito en el análisis de cointegración. Partiendo de un modelo uniecuacional general para la inflación, el proceso general a específico lleva a modelos que son sumamente parsimoniosos e intuitivos y que se desempeñan razonablemente bien en las pruebas estadísticas de diagnóstico y constancia. Se presentan las implicaciones dinámicas del modelo de inflación de precios no controlados. Para ello se derivan dos modelos simples para la tasa de crecimiento salarial y para la brecha de producto.

Los ejercicios llevados a cabo muestran que ajustes a los salarios tienen un efecto inmediato mayor que el del tipo de cambio pero que después de un tiempo el efecto de este último predomina. También se presentan las respuestas a choques de precios públicos y la brecha de producto. Se explora el efecto de ajustes simultáneos del tipo de cambio y los precios precios ante un incremento salarial. Finalmente, se hacen comparaciones con un país de baja inflación (Australia) y se muestra que la velocidad de transmisión de choques del tipo de cambio es sustancialmente mayor en México. 


\section{A Pruebas de Raíces Unitarias}

El cuadro 2 muestra los resultados de las pruebas de raíces unitarias. Las pruebas de Dickey Fuller Aumentada (ADF) se estimaron a partir de 13 rezagos incluyendo una constante y una tendencia. La especificación que aparece en el cuadro 1 resultó la especificación más parsimoniosa. Por contraste, la prueba de Phillips-Perron $(\mathrm{PhP})$ se estima con cuatro rezagos (que es el número implicado por el criterio de Newey-West). En los dos casos decidimos incluir una constante, una tendencia y un cierto número de rezagos dependiendo de las características del proceso y de la significancia estadística de los estimados.

Cuadro 2: Pruebas de Raíces Unitarias

\begin{tabular}{|c|c|c|c|c|}
\hline & \multicolumn{2}{|c|}{ DICKEY FULLER A. } & \multicolumn{2}{|c|}{ PHILLIP-PERRON } \\
\hline & Estadístico & Especificación & Estadístico & Especificación \\
\hline \multicolumn{5}{|c|}{ NIVELES } \\
\hline $\mathrm{p}$ & -2.488 & Const. & 0.133 & Const. $\mathrm{y}$ tend. \\
\hline $\mathbf{y}$ & -2.821 & Const. y tend. & $-3.564^{* *}$ & Const. y tend. \\
\hline $\mathrm{w}$ & -2.519 & Const. & -2.321 & Const. \\
\hline $\mathrm{pp}$ & -2.428 & Const. & -2.569 & Const. y tend \\
\hline epeu & -2.379 & Const. & -0.410 & Const. y tend. \\
\hline$(w-p)$ & $-2.670^{*}$ & Const. & $-6.045^{* * *}$ & Const. \\
\hline (epeu-p) & $-2.795^{*}$ & Const. & -2.099 & Const. \\
\hline pnc & -0.694 & Nada & -2.488 & Const. y tend. \\
\hline \multicolumn{5}{|c|}{ DIFERENCIAS } \\
\hline $\mathrm{p}$ & -2.330 & Const. & $-3.810^{* * *}$ & Const. \\
\hline $\mathbf{y}$ & $-3.411^{* *}$ & Const. & $-28.518^{* * *}$ & Const. \\
\hline w & -0.841 & Nada & $-23.094^{* * *}$ & Nada \\
\hline pp & $-1.619^{*}$ & Const. & $-5.377 * * *$ & Const. \\
\hline epeu & $-2.710^{* * *}$ & Nada & $-10.587 * * *$ & Const. \\
\hline$(w-p)$ & $-2.487^{* *}$ & Nada & $-34.495^{* * *}$ & Nada \\
\hline (epeu-p) & $-5.639 * * *$ & Nada & $-12.254^{* * *}$ & Nada \\
\hline pnc & -1.492 & Nada & $-2.761^{* * *}$ & Const. \\
\hline
\end{tabular}

Por ejemplo, en el caso del (logaritmo del) INPC $(p)$ y el índice de la producción industrial $(y)$ iniciamos ambas pruebas incluyendo constante, tendencia y trece rezagos. A continuación probamos la significancia estadística de los últimos de ellos, la constante y la tendencia y eliminamos en otra corrida los estimados que no pasaron la prueba. Repetimos el proceso hasta que los coeficientes restantes tengan la significancia estadística deseada (5\%).

Para la primera diferencia de las mismas variable se comenzó de nueva cuenta incluyendo 13 rezagos, una constante y omitimos la tendencia (de lo contrario estaríase implicando una tendencia cuadrática muy poco razonable en los niveles). De ahora en adelante utilizaremos el $5 \%$ de significancia como referencia en nuestra discusión de resultados.

Como se puede observar, hay varias contradicciones en los resultados. Primero, en niveles, ambas pruebas sugieren que la mayor parte de las series tienen al menos una raíz unitaria con algunas excepciones. La prueba $\mathrm{PhP}$ sugiere que el índice de la produción industrial es $I(0)$ con una constante y una tendencia 
lineal (aunque el rechazo de la hipótesis de raíz unitaria es marginal) mientras que ADF la clasifica claramente como $I(1)$ con constante y tendencia determinística. En diferencias, todas las series son clasificadas como $I(0)$ por $\mathrm{PhP}$ mientras que ADF considera que la primera diferencia del INPC es $I(1)$.

En resumen, $y$ es a lo sumo $I(1)$ pero podría ser $I(0)$ con una tendencia determinística. El Indice Nacional de Precios al Consumidor, el índice de salarios y los precios externos son a lo sumo I(2) pero podrían ser I(1). El salario real y el tipo de cambio real son a lo sumo $I(1)$ pero podrían ser $I(0)$. En el documento consideramos que ninguna serie es $I(\mathscr{2})$ debido a que las series consideradas son altamente heteroscedásticas y la prueba ADF es muy sensible a tal factor.

\section{B Análisis de Cointegración}

Debido a que la relación de largo plazo que se desea estimar tiene más de dos variables, es natural comenzar con un método de sistema. La mayor parte de la evidencia experimental apunta a que el método de Johansen ( $\mathrm{JOH}$ ) tiene mejor desempeño que otros métodos de sistema disponibles. ${ }^{24}$ Definiendo $\mathbf{Z}_{\mathrm{t}}$ $=\left(p_{t}, w_{t}\right.$, epeu $\left.u_{t}\right)$ como el vector de variables en la relación de largo plazo, el método de Johansen parte de la estimación del siguiente vector autorregresivo de corrección de error:

$$
\Delta \mathbf{Z}_{\mathrm{t}}=\mu+\Pi \mathbf{Z}_{\mathrm{t}-\mathbf{1}}+\sum_{\tau=\mathbf{1}}^{\mathbf{s}} \Xi_{\mathbf{s}} \Delta \mathbf{Z}_{\mathrm{t}-\mathbf{s}}+\Phi X_{t}+\mathbf{u}_{\mathrm{t}}
$$

donde $\mu$ es un vector de constantes de dimensión $3, \Pi=\left[\pi_{i j}\right]$ es la matriz de coeficientes de largo plazo de dimensión $3 \times 3$ y rango $(r)$ menor que tres. Las $\Xi=\left[\xi_{i j}\right]$ son las matrices de coeficientes de corto plazo (de dimensión $3 \times 3$ ) y $u_{t}$ es un vector de términos de error serialmente independientes pero quizás contemporánemente correlacionados. La matriz $\Phi=\left[\phi_{i l}\right]$ de dimensión $3 \times L$ es la matriz de coeficientes del vector de variables exógenas $X_{t}$ de dimensión $L$. Entre las variables exógenas se pueden incluir variables estacionales, indicadores de intervención o algunas variables $I(0)$ que afecten la dinámica de corto plazo del sistema.

Es útil redefinir a la matriz de largo plazo como:

$$
\Pi=\alpha \beta^{\prime}
$$

donde $\alpha=\left[\alpha_{i j}\right]$ y $\beta=\left[\beta_{i j}\right]$ son dos matrices de dimensión $3 \times 3$ y rango $r$. La matriz $\beta$ es la matriz formada por los vectores de cointegración. Pruebas de hipótesis sobre su rango, $r$, determinan el número de vectores de cointegración en el sistema. La matriz $\alpha$ contiene los coeficientes de retroalimentación. Sobre esta última matriz se llevan a cabo las pruebas de exogeneidad débil que se

\footnotetext{
${ }^{24}$ Ver Maddala y Kim (1998) para una extensiva revisión de la literatura al respecto. Debido a que JOH es bien conocido y existen numerosas referencias en la literatura donde se describe en detalle (por ejemplo Johansen (1995) y Hatanaka (1996)), no se mencionará más que los detalles estrictamente indispensables.
} 
reportan más adelante. Si hay cointegración, al menos un elemento de $\alpha$ debe ser significativo. ${ }^{25}$

Los cuadros 3 y 4 que se presentan a continuación resumen el análisis de cointegración llevado a cabo para el índice nacional de precios al consumidor, $p, \mathrm{y}$ el índice de precios no controlados, $p n c$, respectivamente. El análisis en el cuadro 3 comienza en 1980 mientras que en el cuadro 4 inicia en 1985 debido a la disponibilidad de datos. En ambos cuadros un asterisco indica rechazo de la hipótesis nula al $5 \%$ de significancia mientras dos asteriscos señalan rechazo al $1 \%$.

Cuadro 3: Análisis de Cointegración para el Logaritmo del Indice Nacional de Precios al Consumidor, $p$ (1980-1998)

\begin{tabular}{|c|c|c|c|c|}
\hline \multicolumn{5}{|c|}{ (i) Análisis de Cointegración } \\
\hline Eigenvalores & 0.112 & 0.056 & . & 0.024 \\
\hline Hipótesis nula & $\operatorname{ran} .=0$ & $\operatorname{ran} . \leq 1$ & . & $\operatorname{ran} . \leq 2$ \\
\hline$\lambda_{t r a z a}$ & $36.17 * *$ & $14 . \overline{62}$ & . & $4 . \overline{30} *$ \\
\hline Val. Crit. (95\%) & 29.70 & 15.40 & . & 3.80 \\
\hline \multicolumn{5}{|c|}{ (ii) Estandarización del vector de Cointegración y ajuste de los coeficientes } \\
\hline Variable & $\bar{p}$ & $\bar{w}$ & & epeu \\
\hline Vec. Coin. Nor. $\gamma$ & 1.000 & -0.360 & . & -0.630 \\
\hline Ajuste de Coef. $\alpha$ & -0.092 & 0.008 & . & 0.077 \\
\hline \multicolumn{5}{|c|}{ (iii) Pruebas de significancia, exogeneidad débil y exogeneidad fuerte de una variable dada } \\
\hline Variable & $\bar{p}$ & w & & epeu \\
\hline Exclusión $\chi^{2}(1)$ & $11.27^{* *}$ & $6.802^{* *}$ & - & $12.830^{* *}$ \\
\hline Exog. débil $\chi^{2}(1)$ & $8.23^{* *}$ & 0.015 & . & 0.314 \\
\hline Exog. fuerte $\chi^{2}(1)$ & $98.609^{* *}$ & $150.29^{* *}$ & . & 33.272 \\
\hline \multicolumn{5}{|c|}{ (iv) Prueba de Homogeneidad lineal de los coeficientes $\left[\beta_{p}=-\left(\beta_{w}+\beta_{e}\right)\right]$} \\
\hline Homog. lineal $\chi^{2}(1)$ & & 0.126 & & \\
\hline \multicolumn{5}{|c|}{ (v) Especificación de las pruebas } \\
\hline Ecuación & $\mathrm{p}$ & $\overline{\mathrm{w}}$ & epeu & Prueba conjunta \\
\hline $\operatorname{AR}(7,102)$ & $0.45[.870]^{*}$ & $0.20[.999]$ & $0.73[.64]$ & $F(63,436)=0.86[.76]$ \\
\hline Normalidad $\chi^{2}(2)$ & $55.70[.000]^{* *}$ & $13.39[.001]^{* *}$ & $158.27[.00]^{* *}$ & $\chi^{2}(6)=227.86[.00]^{* *}$ \\
\hline Heteroc. $\mathrm{F}(78,90)$ & $1.06[.040]^{*}$ & $1.68[.009]^{* *}$ & $1.02[.46]$ & $\mathrm{F}(468,518)=1.35[.00]^{* *}$ \\
\hline \multicolumn{5}{|c|}{ (vi) Pruebas $F$ para la simplificación de 12 a menos rezagos } \\
\hline $\begin{array}{l}\text { Prueba } F \\
\text { valor } \mathrm{p}\end{array}$ & $\begin{array}{r}12 \text { a } 11 \text { rezagos } \\
{[0.0}\end{array}$ & $\begin{array}{l}(9,406)=2.138^{*} \\
55]\end{array}$ & 12 a 6 reza & $\begin{array}{l}\mathrm{os}(63,499)=1.535^{* *} \\
{[0.0074]}\end{array}$ \\
\hline
\end{tabular}

Los valores de probabilidad van entre paréntesis cuadrados.

Una descripción detallada de las pruebas aquí proporcionadas se puede obtener en Hendry (1995).

\footnotetext{
${ }^{25}$ En tal caso, la variable asociada a alguno de los elementos significativos se dice que rechaza la hipótesis de ser débilmente exógena. Las variables que resultan ser débilmente exógenas admiten ser consideradas como fijas al estimar los parámetros de la ecuación de corto plazo de las variables endógenas.
} 
Cuadro 4: Análisis de Cointegración para el Logaritmo del Indice de Precios No Controlados, pnc (1985-1998)

\begin{tabular}{|c|c|c|c|c|}
\hline \multicolumn{5}{|c|}{ (i) Análisis de Cointegración } \\
\hline Eigenvalores & 0.253 & 0.077 & . & 0.021 \\
\hline Hipótesis nula & $\operatorname{ran} .=0$ & $\operatorname{ran} \cdot \leq 1$ & . & $\operatorname{ran} \cdot \leq 2$ \\
\hline$\lambda_{t r a z a}$ & $47.43^{* *}$ & 12.17 & . & 2.54 \\
\hline Val. Crit. (95\%) & 29.70 & 15.40 & . & 3.80 \\
\hline \multicolumn{5}{|c|}{ (ii) Estandarización del vector de Cointegración y ajuste de los coeficientes } \\
\hline Variable & pnc & $\mathrm{w}$ & & epeu \\
\hline Vec. Coint. Nor. $\gamma$ & 1.000 & -0.451 & . & -0.562 \\
\hline Ajuste de Coef. $\alpha$ & -0.063 & 0.122 & . & -0.016 \\
\hline \multicolumn{5}{|c|}{ (iii) Pruebas de significancia, de exogeneidad débil y exogeneidad fuerte de una variable dada } \\
\hline Variable & pnc & $\mathrm{w}$ & & epeu \\
\hline Exclusión $\chi^{2}(1)$ & $26.88^{* *}$ & $26.32 * *$ & . & $23.13^{* *}$ \\
\hline Exog. débil $\chi^{2}(1)$ & $17.12^{* *}$ & $6.18^{* *}$ & . & 0.03 \\
\hline Exog. fuerte $\chi^{2}(1)$ & $140.74^{* *}$ & $82.92 * *$ & . & 25.821 \\
\hline \multicolumn{5}{|c|}{ (iv) Prueba de Homogeneidad lineal de los coeficientes $\left[\beta_{p}=-\left(\beta_{w}+\beta_{e}\right)\right]$} \\
\hline Homog. lineal $\chi^{2}(1)$ & & 2.59 & & \\
\hline \multicolumn{5}{|c|}{ (v) Especificación de las pruebas } \\
\hline Ecuación & pnc & $\overline{\mathrm{w}}$ & epeu & Prueba conjunta \\
\hline $\operatorname{AR}(7,102)$ & $2.17[.040]^{*}$ & $1.15[.34]$ & $0.37[.92]$ & $\mathrm{F}(63,257)=1.06[.37]$ \\
\hline Normalidad $\chi^{2}(2)$ & $11.85[.002]^{* *}$ & $8.17[.02]^{*}$ & $99.56[.00]^{* *}$ & $\chi^{2}(6)=109.67[.00]^{* *}$ \\
\hline Heteroc. $\mathrm{F}(78,30)$ & $1.72[.048]^{*}$ & $0.97[.55]$ & $0.40[.99]$ & $\mathrm{F}(468,158)=0.64[.99]$ \\
\hline \multicolumn{5}{|c|}{ (vi) Pruebas $F$ para la simplificación de 12 a menos rezagos } \\
\hline Prueba $F$ & 12 a 11 rezagos & $(9,260)=1.20^{*}$ & 12 a $6 \mathrm{r}$ & $\operatorname{agos} F(63,320)=1.45^{*}$ \\
\hline valor $\mathrm{p}$ & {$[0.0$} & & & {$[0.021]$} \\
\hline
\end{tabular}

Los valores de probabilidad van entre paréntesis cuadrados.

Una descripción detallada de las pruebas aquí proporcionadas se puede obtener en Hendry (1995).

La especificación para el vector de cointegración incluyó una constante (de cerca de -6.25) que no se reporta. El primer resultado importante se encuentra en la parte (i) de los cuadros 3 y 4 . De acuerdo al estadístico de la traza, ${ }^{26}$ $\lambda_{t r a z a}$, para ambos sistemas existe un solo vector de cointegración. Esto se puede ver en la primera columna del tercer renglón de dichos cuadros. En el primer cuadro, el estadístico de la traza es de 36.17 , el cual excede por bastante el valor crítico para el $5 \%$ de significancia de la hipótesis de cero vectores de cointegración (o que el rango de la matriz $\beta, r$ es igual a cero).

El rechazo de la misma hipótesis en el segundo cuadro es aún más fuerte. El estadístico de la traza para la hipótesis de que hay a lo sumo un vector de cointegración (o que $r \leq 1$ ) para ambos cuadros (tercer renglón y segunda columna) es inferior al valor crítico correspondiente. La conclusión es que existe un vector de cointegración y solamente uno.

En la parte (ii) de ambos cuadros, los únicos vectores de cointegración de

\footnotetext{
${ }^{26}$ Es posible utilizar un estadístico alternativo, el del valor característico máximo. No lo reportamos porque produce resultados similares y por las razones que se discuten más adelante.
} 
cada cuadro se normalizan para formar una ecuación de precios (es decir, haciendo igual a uno el coeficiente de $p$ o pnc). Estos vectores normalizados son $\left(\beta_{p}, \beta_{w}, \beta_{e}\right)=(1.00,-0.36,-0.63)$ у $\left(\beta_{p n c}, \beta_{w}, \beta_{e}\right)=(1.00,-0.45,-0.56)$. Estos resultados están relacionados con el modelo de la sección 4.1 haciendo que $\gamma_{w}=-\beta_{w}=0.36$ y $\gamma_{e}=-\beta_{e}=0.63$, es decir los componentes del vector de cointegración debidamente normalizados se interpretan como los parámetros de la ecuación 1 . Se observa que los vectores son ligeramente diferentes para el comportamiento del Indice Nacional de Precios al Consumidor y el índice de precios no controlados.

En la parte (iii) de los cuadros se incluyen las pruebas de exclusión de variable (es decir una prueba para decidir si una variable puede ser eliminada de la relación de largo plazo) y las pruebas de exogeneidad débil y exogeneidad fuerte. Las pruebas de exclusión para cada variable son rechazadas fuertemente al $1 \%$. Se concluye que las tres variables consideradas deben estar en la relación de largo plazo.

Las pruebas de exogeneidad débil (es decir, de significancia para los coeficientes de ajuste $\alpha$ ) difieren un poco. Para el sistema que incluye al Indice Nacional de Precios al Consumidor, $p$, esta última variable es la única que rechaza la hipótesis de exogeneidad débil. Por contraste, en el otro sistema, además del índice de precios no controlados $p n c$, la variable salarios, $w$, también rechaza la hipótesis. A pesar de este resultado, que atribuimos a la muestra más corta, en el modelaje de corto plazo procedemos de la misma manera que con el sistema para el Indice Nacional de Precios al Consumidor, $p$.

Las pruebas de exogeneidad fuerte establecen si el pronóstico de una variable puede ser mejorado incorporando información proveniente de las otras variables. En otras palabras, ésta es una prueba de causalidad en el sentido de Granger conjunta para los términos de corto y largo plazo. Por esta razón una variable que rechaza la hipótesis de exogeneidad débil rechazará la de exogeneidad fuerte (la prueba de exogeneidad débil se puede interpretar como una prueba de Granger para el largo plazo únicamente).

En los dos cuadros se puede observar que la única variable fuertemente exógena es el nivel de precios externo. Esto tiene implicaciones importantes. La primera es que no hay evidencia de que la inflación o los salarios ayuden a predecir (o causar en el sentido de Granger) al tipo de cambio y en cambio éste si ayuda a pronosticar a aquéllos. La segunda implicación es que uno puede tomar como variable estrictamente exógena a los precios externos pero no a los salarios para hacer pronósticos y simulaciones. Esto justifica la omisión de un modelo explícito para el tipo de cambio en los ejercicios de simulación de la sección 6.

En la parte (iv) de los dos cuadros se prueba la hipótesis de que el negativo de la suma de los coeficientes para salarios y precios externos es igual al coeficiente del índice de precios correspondiente, esto es:

$$
H_{o}: \beta_{p}=-\left(\beta_{w}+\beta_{e}\right)
$$

En ambos cuadros la hipótesis no se rechaza. Esta prueba de hipótesis es equivalente a probar que la suma de las elasticidades de la ecuación 1 es igual a 
uno $\left(\gamma_{w}+\gamma_{e}=1\right)$ por lo que se concluye que la relación de equilibrio de largo plazo es una ecuación de precios con la propiedad de homogeneidad lineal.

La parte (v) de los cuadros contiene las pruebas de especificación para el vector de corrección de error sobre el que se basa este análisis. El estadístico del multiplicador de Lagrange para detectar autocorrelación no indica la presencia de este problema en ninguna ecuación y esto ocurrió para cualquier longitud de rezagos elegida. La única excepción a esto fue un no rechazo marginal para la ecuación de la inflación de precios no controlados $\Delta p n c$, lo cual se atribuye (y se comprueba más adelante) a una pérdida de poder de la prueba debida al uso de un número elevado de rezagos con una muestra relativamente corta. La prueba de normalidad según el estadístico Jarque-Bera indica que los residuales en todas las ecuaciones son no normales. Finalmente, algunas ecuaciones en cada sistema presentan problemas de heterocedasticidad.

El origen de los problemas de especificación anteriores no es difícil de rastrear. La no normalidad y quizás la heterocedasticidad provienen de la existencia de varias observaciones que podrían considerarse valores extremos en la historia de las series. Con una cantidad suficiente de variables dicótomas se pueden suprimir o paliar tales problemas.

Como una cuestión de principio no utilizamos tal alternativa por las siguientes razones: a) La receta tradicional de utilizar variables dicótomas (dummy) para ajustar valores extremos resulta en muchas ocasiones un problema de especificación más serio que los que trata de resolver. ${ }^{27}$ b) Las pruebas de especificación indican problemas que no son serios. El uso de las pruebas de hipótesis es asintóticamente válido aún en presencia de no normalidad y heteroscedasticidad. $^{28}$ En cualquier caso, para tratar con valores extremos hay alternativas mejores que el uso de variables dicótomas ${ }^{29}$ cuya aplicación hemos dejado para una investigación futura.

\section{Análisis de la Constancia del Modelo de Cor- to Plazo}

Una vez que se ha mostrado el buen desempeño de los modelos de corto plazo en las pruebas de significancia y diagnóstico, el siguiente paso es examinar la esta-

\footnotetext{
${ }^{27}$ Por ejemplo, el modelar eventos intrínsicamente estocásticos como eventos determinísticos es obviamente un problema mayor. Además, el uso para pronóstico o simulación de modelos con variables dicótomas es también cuestionable.

${ }^{28}$ Aunque las muestras no son suficientemente grandes como para aplicar argumentos asintóticos, uno puede acudir a la evidencia Montecarlo para muestras pequeñas, encontrada en la literatura (Cheung and Lai (1993)) y ésta sugiere que el estadístico de la traza es más robusto para probar cointegración que el del valor característico máximo (por esta razón la discusión se concentró en él). Además, si bien es verdad que hay un sesgo del método de Johansen a encontrar cointegración espuria en presencia de no-normalidad, con un método alternativo que es más robusto ante este problema encontramos resultados similares: estimamos el vector de cointegración con FM-OLS y después construimos un modelo de corrección de error donde la hipótesis de cointegración no fue rechazada. La hipótesis de homogeneidad lineal tampoco se puede rechazar.

${ }^{29}$ Como los métodos robustos de Franses y Lucas (1998).
} 
bilidad de éstos durante el período considerado. Esto es crucial para cualquier modelo que quiera usarse en pronósticos o análisis de política económica. ${ }^{30}$

Los modelos descritos parecen ser razonablemente constantes a través del período, especialmente considerando la serie de cambios de política económica y choques que la economía mexicana ha experimentado. Esto es importante debido a que el estimar un modelo para el período de tipo de cambio flexible, por ejemplo, es poco factible por el corto período de tiempo en que ha estado en función. Por ello, estos modelos son buenos candidatos para ser usados en la evaluación de política monetaria. En la gráfica 10 se presentan las trayectorias de la suma de errores al cuadrado, los residuales, estimados de parámetros recursivos y de la secuencia de estadísticos de Chow para puntos de quiebre del modelo para la inflación general, $d p$.
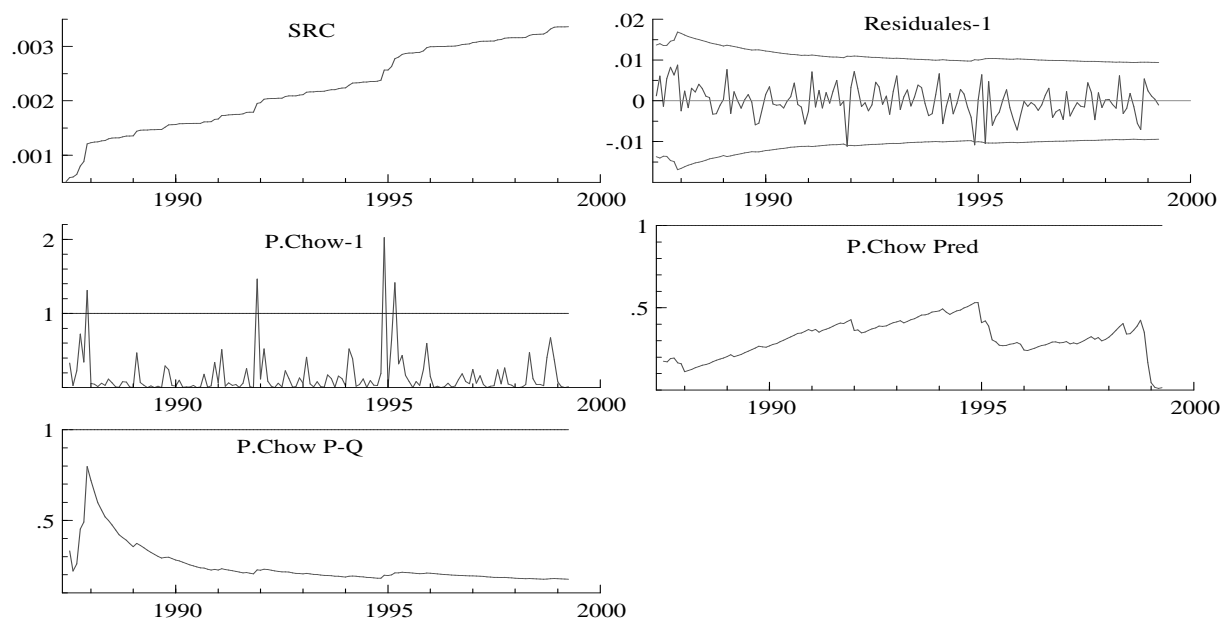

Gráfica 10: Pruebas de Estabilidad del Modelo.

El primer panel de la gráfica 10 muestra cómo cambia la suma de los residuales al cuadrado al aumentar el tamaño de la muestra. Idealmente, si el modelo es estable, la línea debería aproximarse a una recta, lo que ocurre en este caso. Un modelo constante requiere que estos residuales se encuentren comprendidos

\footnotetext{
${ }^{30}$ Engle y Hendry (1993) discuten el concepto de super-exogeneidad como un requisito para modelos econométricos que legítimamente puedan usarse en la evaluación de la política económica aún sin incorporar expectativas y comportamiento optimizador. La idea es que si un modelo econométrico cumple con las condiciones de a) exogeneidad débil de las variables del lado derecho; b) constancia de los parámetros y; c) invarianza del modelo ante un conjunto determinado de intervenciones, entonces el modelo puede usarse legítimamente en la evaluación de política económica pues la Crítica de Lucas no le afecta empíricamente.
} 
en las bandas de confianza del 5\%. El segundo panel muestra la trayectoria de residuales recursivos junto con las respectivas bandas de confianza de $\mathbf{9 5 \%}$. Es bastante claro que sólo en tres ocasiones (dos de ellas a principios de 1995) dicha trayectoria se acerca a la parte inferior de la banda.

Los siguientes paneles muestran la trayectoria de los estadísticos de Chow, que identifican inestabilidad o posibles cambios estructurales acaecidos en fechas inciertas. Estos, al permanecer dentro de las líneas de confianza del 5\% (normalizadas a 1), demuestran que el modelo es constante durante el período de estimación. Solamente la tercera gráfica tiene puntos que se salen de la línea de significancia (los tres últimos coincidiendo con los puntos problema del segundo panel).

Las gráficas de coeficientes recursivos muestran el valor de los estimados de los parámetros conforme el tamaño de la muestra aumenta. Un modelo constante requiere que estos estimados no cambien al variar la longitud de la muestra. Las trayectorias de estos coeficientes se presentan en la gráfica 11, donde se comprueba la constancia de la mayoría de ellos, con la excepción aparente del coeficiente de corto plazo para la inflación importada. Para este último se puede observar un desplazamiento brusco al comienzo de 1995, precisamente cuando se adoptó el régimen de tipo de cambio flexible. Esto ocurrió simultáneamente a una serie de fuertes movimientos en la paridad los cuales podrían explicar este comportamiento.
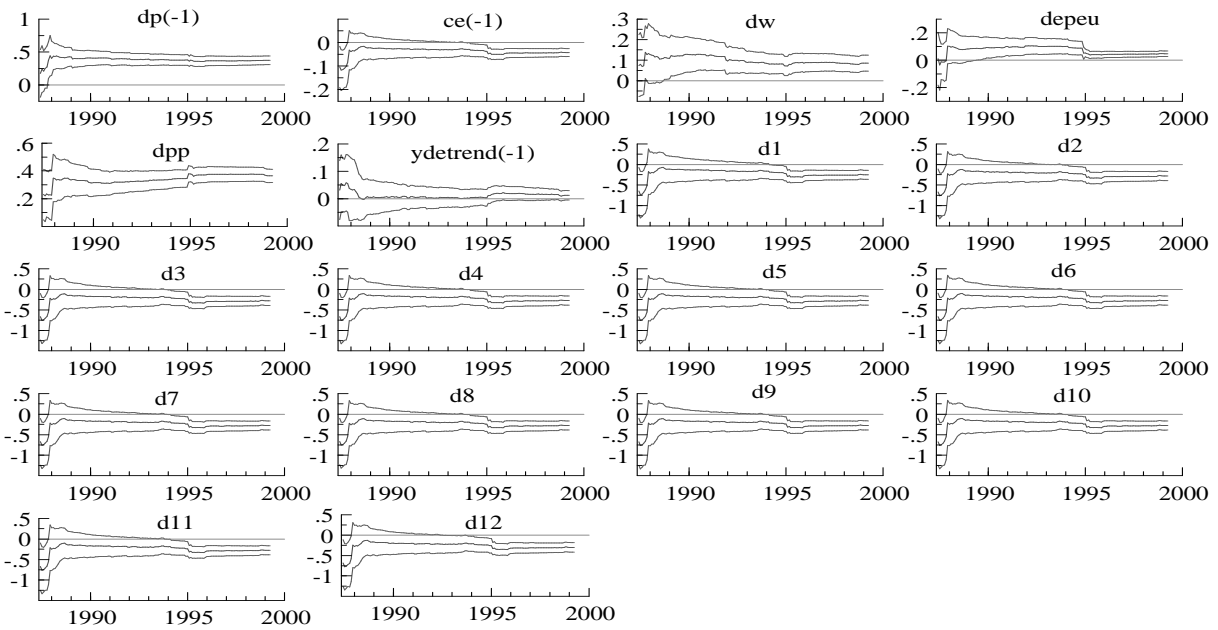

Gráfica 11: Estimados recursivos de los parámetros.

Para investigar si el cambio brusco en dicho coeficiente es estadísticamente 
significativo, corrimos la misma regresión con un término de interacción adicional (construído con una variable indicador que es cero hasta 1994 multiplicada por la inflación importada contemporánea). El estimado para el coeficiente de este nuevo término tuvo el signo esperado (negativo) pero no fue significativo. De hecho, la prueba de constancia de estabilidad individual (no reportada) sólo es no significativa para tres coeficientes de las variables estacionales.

\section{Un Modelo para los Salarios}

El modelo de inflación se formuló bajo el supuesto, respaldado por las pruebas de exogeneidad débil, de que no era necesario conocer los parámetros de la ecuación de salarios ni de precios importados. En otras palabras, estimamos un modelo condicional de inflación. Sin embargo, es muy importante tener un modelo estadístico de salarios si se quiere utilizar la ecuación de inflación con fines de pronóstico o simulación.

\section{D.1 Formulación del Modelo de Salarios}

En esta sección se desarrolla un modelo para caracterizar el comportamiento de los salarios que complementa en parte al modelo de inflación de la sección previa. Este modelo será útil para utilizar nuestra ecuación en ejercicios de pronóstico y simulación. ${ }^{31}$

Como se mostró en el apéndice $\mathrm{B}$, los salarios son una variable débilmente exógena. Por ello el término de corrección de error no debe aparecer entre las variables explicatorias. Este resultado se comprobó introduciendo tal término en varias especificaciones de la ecuación de salarios y en todas ellas no se encontraron efectos significativos.

Aplicando una vez más el procedimiento estándar de ir de lo "general a lo específico," llegamos a los dos modelos dinámicos para los salarios contractuales presentados en el cuadro 5. Principalmente se discutirá el primero, que parece ser el más aceptable en términos estadísticos, debido a las pruebas de diagnóstico.

\section{D.2 Interpretación de los Parámetros}

Primero hay que mencionar que la estimación para la primera ecuación si fue posible llevarla a cabo desde 1980. Hacerlo desde 1985 no cambia los resultados demasiado puesto que, como se verá, este modelo es extremadamente estable. El segundo modelo se estimó desde 1985 debido a que, como se apuntó antes, las series de precios de bienes públicos comienza en ese año.

Las primeras variables que se incluyeron en la ecuación preferida son los rezagos uno, tres y doce de la tasa de cambio de los salarios. De entre estos

\footnotetext{
${ }^{31}$ Este es un modelo preliminar que posiblemente pueda mejorarse en varios aspectos. Por ejemplo, otras variables podrían entrar en la explicación de la dinámica salarial o los factores estacionales podrían ser modelados de modo diferente.
} 
Cuadro 5: Modelos de Salarios

\begin{tabular}{|c|c|c|}
\hline VARIABLES & MODELO 1 & MODELO 2 \\
\hline$d w_{t-1}$ & $\begin{array}{cc}-0 & .1363 \\
(0 & .0833)\end{array}$ & $\begin{array}{ll}-0 & .2423 \\
(0 & .0705)\end{array}$ \\
\hline$d w_{t-3}$ & $\begin{array}{c}0.0288 \\
(0.0229)\end{array}$ & $\begin{array}{c}0.0361 \\
(0.0161)\end{array}$ \\
\hline$d w_{t-12}$ & $\begin{array}{c}0.3199 \\
(0.0663)\end{array}$ & $\begin{array}{c}0.3046 \\
(0.0611)\end{array}$ \\
\hline$d p_{t-1}$ & $\begin{array}{c}0.4995 \\
(0.2521)\end{array}$ & . \\
\hline$d p_{t-2}$ & $\begin{array}{c}0.3289 \\
(0.2495)\end{array}$ & . \\
\hline$d p n c_{t-1}$ & . & $\begin{array}{c}0.6715 \\
(0.1742)\end{array}$ \\
\hline$d p p_{t-1}$ & . & $\begin{array}{c}0.3867 \\
(0.1112)\end{array}$ \\
\hline ydetrend & $\begin{array}{c}0.0765 \\
(0.0353)\end{array}$ & $\begin{array}{c}0.0763 \\
(0.0300)\end{array}$ \\
\hline$R^{2}$ & 0.9629 & 0.9803 \\
\hline$R^{2}$ Ajustada & 0.9614 & 0.9793 \\
\hline E.E. Regresión & 0.0255 & 0.0190 \\
\hline Crit. Akaike & -7.2935 & -7.8756 \\
\hline Crit. Schwartz & -7.1482 & -7.7004 \\
\hline Coef. Asimetrí & 0.2573 & 1.0106 \\
\hline Curtosis & 5.3464 & 6.8546 \\
\hline LM (12) Autoc. & 0.9578 & $2.03394^{* *}$ \\
\hline Est. Jarque-Bera & $49.5316^{* * * *}$ & $123.9187^{* * *}$ \\
\hline $\mathrm{LM}$ (12)ARCH & $5.6503^{* * * *}$ & $4.4045^{* * *}$ \\
\hline Prueba de White & $5.8055^{* * * *}$ & $2.8213^{* * * *}$ \\
\hline Prueba RESET (1) & $9.1893^{* * *}$ & $4.2026^{* *}$ \\
\hline
\end{tabular}

tres, el último es el más fuerte y significativo. Esto tiene sentido dado que una gran cantidad de contratos se negocian exactamente año tras año. Los rezagos primero y tercero no son muy significativos pero su presencia es necesaria para evitar correlación serial.

Sin embargo, el efecto más fuerte proviene de la historia inflacionaria, representada aquí por los dos primeros rezagos de esta variable. Esto de nueva cuenta tiene mucho sentido económico. El efecto total de la inflación pasada sobre la tasa de cambio de los salarios es de 0.76, es decir, aproximadamente tres cuartas partes de la inflación pasada se incorporan a los salarios actuales.

El coeficiente de la brecha de producto contemporánea es también significativo y tiene el signo esperado. Por cada punto porcentual de desviación de la actividad económica de su tendencia, la tasa de salarios se incrementa en 0.08. Notamos que esta variable ayuda a mejorar la estabilidad del modelo.

El segundo modelo descompone los efectos de la inflación en aquéllos provenientes de los bienes no controlados que resultan de los bienes públicos. $\mathrm{Al}$ parecer la inflación de los precios no controlados tiene un efecto mayor al de la inflación de bienes administrados (0.67 contra 0.39). Sin embargo, es importante recordar que los bienes públicos tienen una participación promedio de 
apenas $17 \%$ en la canasta de bienes sobre la que se elabora el INPC. Así que, en términos relativos, el efecto de los bienes públicos sobre los salarios es mayor si ajustamos por dicha participación. Una forma simple de hacerlo es dividir las respectivas elasticidades entre las participaciones en el INPC. Claramente, el efecto ponderado de los bienes públicos (igual a $0.39 / 0.17=2.29$ ) es mayor que el de los no controlados (que es $0.67 / 0.83=0.81$ ).

Un conjunto adicional de variables tales como la tasa de crecimiento del salario mínimo, la inflación importada y los agregados monetarios no mostraron poder explicativo y por ello fueron dejadas fuera de la especificación final.

\section{D.3 Pruebas de Diagnóstico}

El segundo modelo tiene un coeficiente de determinación múltiple ajustado mayor al del primero y lo domina en términos de los dos criterios de información (Akaike y Schwartz). Sin embargo, el primero tiene mejores propiedades estadísticas. No rechaza la hipótesis de normalidad de los residuales mientras que el segundo si. Las razones de este rechazo son tanto asimetría como exceso de curtosis. También es notable que en el segundo modelo se presente alguna evidencia de correlación serial (de acuerdo al correlograma, en los rezagos tres y cinco). Los dos modelos tienen problemas de heterocedasticidad, como lo muestran las pruebas ARCH y de White. Ninguna pasa la prueba de RESET. De nueva cuenta, pensamos que la heterocedasticidad es responsable de este rechazo. Esto deja abierta la posibilidad de que haya otros modelos mejores.

\section{Referencias}

[1] Clarida, R. H. y Mark P. Taylor (1997) "The Term Structure of Forward Exchange Premiums and the Forecastability of Spot Exchange Rates: Correcting the Errors", Review of Economics and Statistics, Vol. 79, No. 3, August.

[2] Chen, Y. y K. Lai (1993), "Finite Sample Sizes of Johansen's Likelihood Ratio Tests for Cointegration," Oxford Bulletin of Economics and Statistics, $55,313-328$.

[3] Christoffersen, P. F. y Francis X. Diebold (1997) "Cointegration and LongHorizon Forecasting," NBER Working Paper No. T0217, October 1997.

[4] Crawford, Allan y Marcel Kasumovich (1996) "Does Inflation Uncertainty Vary with the Level of Inflation?" Working Paper 96-9, Bank of Canada, August 1996.

[5] Davidson, J.E.H., D.F. Hendry, F. Srba, y S. Yeo (1978) "Econometric Modelling of the Aggregate Time-Series Relationship Between Consumer's Expenditure and Income in the United Kingdom," Economic Journal, 88, 661-692. 
[6] De Brouwer, Gordon y Neil R. Ericsson (1998) "Modelling Inflation in Australia," Journal of Business \& Economic Statistics, Octubre 1998, Vol. 16, No. 4.

[7] Durevall, Dick (1998) "The Dynamics of Chronic Inflation in Brazil, 19681985," Journal of Business and Economic Statistics, vol. 16, No. 4, Octubre.

[8] Engle, Robert, David F. Hendry (1993) "Testing Super Exogeneity and Invariance in Regression Models," Journal of Econometrics, 56, 119-139.

[9] Franses, Phillip H. y Lucas, A. (1998) "Outlier Detection in Cointegration Analysis," Journal of Business \& Economic Statistics, Vol. 16, No. 4, Octubre.

[10] Gabriel, Vasco J,C.R., Artur C.B. da Silva Lopez y Luis M.R.C. Nunes, (1999) "Instability in Cointegration Regressions: Further Discussion with an Application to Money Demand in Portugal," Instituto Superior de Economia, Universidade Técnica de Lisboa, Documento de Trabalho No. 3-99.

[11] Gamboa, R. (1997) "Efecto de los Precios Administrados Sobre la Inflación," Documentos de Investigación, Banco de México.

[12] Garcia-Ferrer, A. y A. Novales (1998) "Forecasting with Money Demand Functions: The UK Case," Journal of Forecasting, 17, 125-145.

[13] Granger, C.W.J. (1981), "Some Properties of Time Series Data and their Use in Econometric Model Specification," 16, 121-130.

[14] Gregory, A.W. y Hansen, B.E. (1996). "Residual-based Tests for Cointegration in Models with Regime Shifts,"

[15] Hansen, B.E. (1992) "Tests for Parameter Instability in Regressions with I(1) Processes," Journal of Business and Economic Statistics, 10, 321-335.

[16] Hao, K. (1996) "Testing for Structural Change in Cointegrated Regression Models: Some Comparisons and Generalizations," Econometric Reviews, 15, 401-429.

[17] Hatanaka,M. (1996) "Time-Series-Based Econometrics," Oxford University Press.

[18] Hendry, D.F. (1995) "Dynamic Econometrics," Oxford University Press.

[19] Hodrick, R.J. y E.C. Prescott (1997) "Postwar U.S. Business Cycles: An Empirical Investigation," Journal of Money, Credit, and Banking, 29, 1-16.

[20] Johansen, S. (1995) "Likelihood-based Inference in Cointegrated Vector Autoregressive Models," Oxford University Press.

[21] Juselius, Katarina (1992) "Domestic and Foreign Effects on Prices in an Open Economy: The Case of Denmark," Journal of Policy Modeling, 14(4):401-428. 
[22] Khamis, May and Alfredo M. Leone (1999) "Can Currency Demand be Stable Under a Financial Crisis? The Case of México," IMF Working Paper, January.

[23] Maddala G. S., In-Moo Kim (1998) "Unit Roots, Cointegration and Structural Change", Cambridge Univeristy Press.

[24] Magendzo, Igal (1998) "Inflación e Incertidumbre Inflacionaria en Chile," Economía Chilena, v.1, No. 1, agosto.

[25] Mehra, Y.P. "Wage Growth and the Inflation Process: An Empirical Note," American Economic Review, 81, 931-937.

[26] Pérez-López, Alejandro (1996) "Un Estudio Economtrico Sobre la Inflacin en Mxico." Documentos de Investigación, Banco de México, Julio, 1996

[27] Phillips, P.C.B. y Bruce E. Hansen (1990), "Statistical Inference in Instrumental Variables Regression With I(1) Processes," Review of Economic Studies, 57, 99-125.

[28] Quintos, C.E. y Peter C.B. Phillips (1993) "Parameter Constancy in Cointegrating Regressions," Empirical Economics, 18, 675-706.

[29] Rao, B.B. (1994) 'Cointegration”, St. Martin's Press.

[30] Saikkonen, P. (1992) "Estimation and testing of cointegrated systems by an autorregressive approximation". Econometric Theory, 8, 1-27. 